\title{
Convergence of equilibria for bending-torsion models of rods with inhomogeneities
}

\author{
Matthäus Pawelczyk \\ FB Mathematik, TU Dresden \\ 01062 Dresden (Germany)
}

\begin{abstract}
We prove that, in the limit of vanishing thickness, equilibrium configurations of inhomogeneous, three-dimensional non-linearly elastic rods converge to equilibrium configurations of the variational limit theory. More precisely, we show that, as $h \searrow 0$, stationary points of the energy $E^{h}$, for a rod $\Omega_{h} \subset \mathbb{R}^{3}$ with cross-sectional diameter $h$, subconverge to stationary points of the $\Gamma$-limit of $E^{h}$, provided that the bending energy of the sequence scales appropriately. This generalizes earlier results for homogeneous materials to the case of materials with (not necessarily periodic) inhomogeneities.
\end{abstract}

Keywords: elasticity, dimension reduction, homogenization, convergence of equilibria

2000 Mathematics Subject Classification: 74K10, 74B20, 74G10, 74E30, 74Q05

\section{Introduction}

The derivation of asymptotic models for two or three-dimensional elastic objects by lower-dimensional models has a long history, going back as far as to Bernoulli 3 and Euler [7. Both considered thin rods, but starting from a two-dimensional model instead of the three-dimensional one, as we study here. Since then a multitude of such models has been proposed, some incompatible with each other. They usually depend on strong a priori assumptions. An in-depth study of the early history can be found in [13].

We start with the nonlinear three-dimensional model: Let $\Omega_{h} \subset \mathbb{R}^{3}$ be the reference configuration of a thin elastic body, with 'thickness' $h>0$. The stored elastic energy of a deformation $y: \Omega_{h} \rightarrow \mathbb{R}^{3}$ is then given by

$$
E^{h}(y):=\int_{\Omega_{h}} W(\nabla y(x)) \mathrm{d} x
$$


where $W$ is the elastic energy density; typical assumptions on $W$ are similar to those provided in (M1) (M3). One is interested in the limiting behaviour of $E^{h}$ as $h \searrow 0$. One of the first results in terms of $\Gamma$-convergence were for $\Omega_{h}:=\omega \times(-h, h)$ with $\omega \subset \mathbb{R}^{2}$. Roughly speaking $\Gamma$-convergence is equivalent to the convergence of global minimizers $y^{h}$ of $E^{h}$, possibly perturbed by some forcing term, to global minimizers of some limiting energy. For example in 12 the theory for membranes, i.e., the limit for $h^{-1} E^{h}$ was obtained, in [8] the bending theory for plates, i.e., for $h^{-3} E^{h}$. The latter result contains, as a particular case, the model proposed by Bernoulli and Euler. Further scalings $h^{-\alpha} E^{h}$ were later studied in [9]. In this present paper we study rods with small cross-sectional diameter. So in our case the reference configuration is $\Omega_{h}:=(0, L) \times h \omega$ for some $L>0$ and $\omega \subset \mathbb{R}^{2}$. The bending-torsion theory for rods, i.e., the $\Gamma$-limit for $h^{-3} E^{h}$ was obtained by [17]. Under the additional assumption of a linear stress growth, the result was strengthened in 15 by proving that also stationary points $y^{h}$ of $E^{h}$ subconverge to stationary points of the $\Gamma$-limit. All the previous mentioned results were obtained in the case of a single, homogeneous material. In [18] the first $\Gamma$-convergence result for a rod in this regime, i.e. $h^{-3} E^{h}$, with inhomogeneities was proved. This was done under the assumption that the inhomogeneity was periodic, rapidly oscillating and only depending on the 'in-plane' variable $x_{1} \in(0, L)$. All these additional assumptions can be dropped, as was shown in [14. In the present paper we extend the result of [14] by showing that also stationary points subconverge to stationary points of the $\Gamma$-limit.

In [5] the more linear case of $h^{-5} E^{h}$, called the von Kármán model, was studied, and $\Gamma$-convergence and convergence of stationary points was proved. This result, and the one presented here, heavily depend on methods developed in [14, 19].

Now we turn to the precise mathematical description. Let $L>0$ and let $\omega \subset \mathbb{R}^{2}$ be open, bounded, connected. The (scaled) energy of a non-homogeneous rod with length $L$ and cross-section $h \omega$ and external forces $g \in L^{2}\left((0, L), \mathbb{R}^{3}\right)$, deformed by $y:[0, L] \times h \omega \rightarrow \mathbb{R}^{3}$, is given by

$$
\widetilde{\mathcal{E}}^{h}(y)=\frac{1}{h^{4}} \int_{[0, L] \times h \omega} W^{h}\left(\left(x_{1}, h^{-1} x^{\prime}\right), \nabla y(x)\right) \mathrm{d} x-\frac{1}{h^{2}} \int_{[0, L] \times h \omega} g\left(x_{1}\right) \cdot y(x) \mathrm{d} x .
$$

The hypotheses on the elastic energy density $W^{h}:[0, L] \times \omega \times \mathbb{R}^{3 \times 3} \rightarrow[0, \infty)$ are listed in Section 2.2. After performing the usual change of variables $\left(x_{1}, x_{2}, x_{3}\right) \mapsto$ $\left(x_{1}, h x_{2}, h x_{3}\right)$ the $\operatorname{rod} \Omega_{h}$ scales to $\Omega:=\Omega_{1}$ and we obtain

$$
\mathcal{E}^{h}(y)=\frac{1}{h^{2}} \int_{[0, L] \times \omega} W^{h}\left(x, \nabla_{h} y(x)\right) \mathrm{d} x-\int_{[0, L] \times \omega} g\left(x_{1}\right) \cdot y(x) \mathrm{d} x,
$$

where $\nabla_{h}=\left(\partial_{1}, \frac{1}{h} \partial_{2}, \frac{1}{h} \partial_{3}\right)$. As already mentioned, in [14] the $\Gamma$-convergence of $\mathcal{E}^{h}$ along a subsequence to a limiting functional $\mathcal{E}^{0}$ was proved. This limit is given by

$$
\mathcal{E}^{0}\left(y, d_{2}, d_{3}\right):= \begin{cases}\int_{0}^{L} Q_{1}^{0}\left(x_{1}, R^{T}\left(x_{1}\right) R^{\prime}\left(x_{1}\right)\right)-g\left(x_{1}\right) \cdot y\left(x_{1}\right) \mathrm{d} x_{1} & \text { if }\left(y, d_{2}, d_{3}\right) \in \mathcal{A}, \\ \infty & \text { else },\end{cases}
$$


where $Q_{1}^{0}$ is a quadratic form in the second argument, which will be introduced in Proposition 2.3. the class of limiting deformations $\mathcal{A}$ is given by

$$
\begin{aligned}
\mathcal{A}:=\left\{\left(y, d_{2}, d_{3}\right) \in W^{2,2}\left((0, L), \mathbb{R}^{3}\right) \times W^{1,2}\left((0, L), \mathbb{R}^{3}\right)\right. & \times W^{1,2}\left((0, L), \mathbb{R}^{3}\right): \\
\left(y^{\prime}, d_{2}, d_{3}\right) & \left.\in W^{1,2}((0, L), \operatorname{SO}(3))\right\},
\end{aligned}
$$

equipped with the strong $W^{2,2} \times W^{1,2} \times W^{1,2}$-topology, and $R=\left(y^{\prime}, d_{2}, d_{3}\right)$ is the rotation associated with $\left(y, d_{2}, d_{3}\right)$.

Formally the first variation of the energy functional $\mathcal{E}^{h}$ in direction of some test function $\psi:[0, L] \times \omega \rightarrow \mathbb{R}^{3}$ is given by

$$
D \mathcal{E}^{h}(y)[\psi]:=\frac{1}{h^{2}} \int_{[0, L] \times \omega} D W^{h}\left(x, \nabla_{h} y(x)\right): \nabla_{h} \psi \mathrm{d} x-\int_{[0, L] \times \omega} g\left(x_{1}\right) \cdot \psi(x) \mathrm{d} x .
$$

For the first integral to be well-defined, however, we need to impose linear stress growth, i.e., for any $F \in \mathbb{R}^{3 \times 3}$ we require the inequality $\left|D W^{h}(\cdot, F)\right| \leq L(|F|+1)$ to hold. Deformations $y$ satisfying $D \mathcal{E}^{h}(y)[\psi]=0$ for all test functions $\psi$ are said to be stationary. If we impose the boundary condition $y\left(0, x^{\prime}\right)=\left(0, h x^{\prime}\right)$ then the natural class of test functions $\psi$ in (44) are $C^{\infty}\left(\bar{\Omega}, \mathbb{R}^{3}\right)$ maps, which vanish at $\{0\} \times \omega$; we denote this class by $C_{\text {bdy }}^{\infty}\left(\bar{\Omega}, \mathbb{R}^{3}\right)$. Another notion of stationary points exists, introduced by J. Ball in [1], which does not need linear stress growth, and is furthermore compatible with physical growth, i.e., $W(F) \rightarrow \infty$ if $\operatorname{det} F \searrow 0$ and $W(F)=\infty$ if $\operatorname{det} F \leq 0$. In $[\underline{6}$ the convergence of such stationary points for the von Kármán rod (for homogeneous materials) was shown. Due to the highly inhomogeneous material we will need to stay in the first setting. Regardless of the notion of stationarity, and even for homogeneous materials, the existence of stationary points is a subtle issue, see [2, section 2.2, section 2.7].

For $\alpha, \beta, M$ positive constants with $\alpha \leq \beta$ we denote by $\mathcal{W}(\alpha, \beta, M)$ the set of admissible density functions $W^{h}$; the precise definition of the class $\mathcal{W}(\alpha, \beta, M)$ is given by (S1) (S3) below. We can now state the main result of this paper:

Theorem 1.1. Let $\left(W^{h}\right) \subset \mathcal{W}(\alpha, \beta, M), g \in L^{2}\left((0, L), \mathbb{R}^{3}\right)$ and $\left(y^{h}\right) \subset W^{1,2}\left(\Omega, \mathbb{R}^{3}\right)$, such that $y^{h}\left(0, x_{2}, x_{3}\right)=\left(0, h x_{2}, h x_{3}\right)$ for any $h>0$, and furthermore

$$
\limsup _{h \searrow 0} \frac{1}{h^{2}} \int_{\Omega} W^{h}\left(x, \nabla_{h} y^{h}(x)\right) \mathrm{d} x<\infty .
$$

Assume in addition, that each $y^{h}$ is a stationary point of $\mathcal{E}^{h}$, given in (1), subject to natural boundary conditions, i.e., $D \mathcal{E}^{h}\left[y^{h}\right][\psi]=0$ for all $\psi \in C_{\mathrm{bdy}}^{\infty}\left(\bar{\Omega}, \mathbb{R}^{3}\right)$. Then there exists $\left(\bar{y}, \bar{d}_{2}, \bar{d}_{3}\right) \in \mathcal{A}$, such that, up to a subsequence, $y^{h} \rightarrow \bar{y}$ strongly in $W^{1,2}\left(\Omega, \mathbb{R}^{3}\right)$ as $h \searrow 0$, and

$$
\nabla_{h} y^{h} \rightarrow\left(\bar{y}^{\prime}, \bar{d}_{2}, \bar{d}_{3}\right) \quad \text { strongly in } L^{2}\left(\Omega, \mathbb{R}^{3 \times 3}\right) .
$$

Furthermore $\bar{y}(0)=0, \bar{d}_{k}(0)=e_{k}$ for $k=2,3$, and $\left(\bar{y}, \bar{d}_{2}, \bar{d}_{3}\right)$ is a stationary point of $\mathcal{E}^{0}$, where $\mathcal{E}^{0}$ is given in (2). 
Remark. It is easily seen that there are sequences $\left(y^{h}\right)$ satisfying the boundary conditions $y^{h}\left(0, x_{2}, x_{3}\right)=h x_{2} e_{2}+h x_{3} e_{3}$ such that (5) holds. Thus an application of Poincaré's inequality shows that (5) holds automatically for a minimizing sequence $\left(y^{h}\right)$.

Remark. The theorem also holds true for the more general forces $\widetilde{g} \in L^{2}\left(\Omega, \mathbb{R}^{3}\right)$. For this the forces in the limiting energy must be replaced by the mean of $\widetilde{g}$ on $\omega$, i.e., by $\int_{\omega} g\left(\cdot, x^{\prime}\right) \mathrm{d} x^{\prime}$. The more general statement can be proved identically, up to a few additional error terms, but which converge trivially to zero for $h \searrow 0$.

The proof of Theorem 1.1 is split into two main parts. For the first one we follow closely the paper [15], where the corresponding result for the homogeneous rod was proved. Their methods for studying the stress can also be applied, with minor modifications, in the more general case considered here. Furthermore we use additional cancellation effects, which simplifies parts of their proof. To conclude their proof they exploit an explicit, linear relationship between the limiting stress and strain, which allows to easily identify the limit equations. In the inhomogeneous case addressed here, such a relationship is less clear and the identification of the limit equation is more involved. Thus for the second part we apply results and methods developed in [5] to identify the limit equation and conclude the proof.

\section{Preliminaries}

\subsection{Notation}

Let $x=\left(x_{1}, x^{\prime}\right) \in \mathbb{R}^{3}$, and let $\mathfrak{p}(x)=\left(0, x^{\prime}\right) \in \mathbb{R}^{3}$ be the projection of $x$ onto $\{0\} \times \omega$. Let $\left(e_{i}\right)_{i=1}^{3}$ be the standard basis of $\mathbb{R}^{3}$. By $(\cdot)$ we denote the inner product on $\mathbb{R}^{3}$ and by (:) the inner product on $\mathbb{R}^{3 \times 3}$, i.e., $A: B=\operatorname{tr}\left(A^{T} B\right)$ for any $A, B \in \mathbb{R}^{3 \times 3}$, with tr being the trace. The twist function $\mathrm{t}: L^{1}(\Omega)^{2} \rightarrow L^{1}(0, L)$ is given by $\mathrm{t}(\phi, \psi)\left(x_{1}\right)=$ $\int_{\omega} x_{3} \phi\left(x_{1}, x^{\prime}\right)-x_{2} \psi\left(x_{1}, x^{\prime}\right) \mathrm{d} x^{\prime}$. We denote by $\iota: \mathbb{R}^{3} \rightarrow \mathbb{R}^{3 \times 3}$ the natural inclusion $\iota(v)=v \otimes e_{1}$, by axl $: \mathbb{R}_{\text {skew }}^{3 \times 3} \rightarrow \mathbb{R}^{3}$ the axial vector $\operatorname{axl}(A)=\left(-A_{23}, A_{13},-A_{12}\right)$ and by $\operatorname{id}_{3 \times 3}$ we denote the $3 \times 3$ identity matrix. By ()$^{\prime}$ we denote the derivative with respect to $x_{1}$, by $\nabla=\left(\partial_{1}, \partial_{2}, \partial_{3}\right)$ the gradient with respect to $x$ and for every $h>0$ we define the scaled gradient as $\nabla_{h}=\left(\partial_{1}, \frac{1}{h} \partial_{2}, \frac{1}{h} \partial_{3}\right)$. For the test functions we define $C_{\mathrm{bdy}}^{\infty}([0, L])=\left\{f \in C^{\infty}([0, L]) \mid f(0)=0\right\}$ and $C_{\mathrm{bdy}}^{\infty}(\bar{\Omega}):=C_{\mathrm{bdy}}^{\infty}\left([0, L], C^{\infty}(\bar{\omega})\right)$, and $W_{\text {bdy }}^{1,2}([0, L])=\left\{f \in W^{1,2}([0, L]) \mid f(0)=0\right\}$ and $W_{\text {bdy }}^{1,2}(\Omega)=W_{\text {bdy }}^{1,2}\left([0, L], W^{1,2}(\omega)\right)$.

\subsection{The nonlinear bending-torsion theory for beams}

Let $L>0$ and let $\omega \subset \mathbb{R}^{2}$ be an open, bounded, connected Lipschitz-domain with $\mathcal{L}^{2}(\omega)=1$ and which is centered, i.e.,

$$
\int_{\omega} x_{2} x_{3} \mathrm{~d} x_{2} \mathrm{~d} x_{3}=\int_{\omega} x_{2} \mathrm{~d} x_{2} \mathrm{~d} x_{3}=\int_{\omega} x_{3} \mathrm{~d} x_{2} \mathrm{~d} x_{3}=0 .
$$

The reference domain $\Omega$ is given by $\Omega=(0, L) \times \omega$. The assumption on the elastic energy density $W$ are as follows: 
Let $\alpha, \beta, M$ be positive constants with $\alpha \leq \beta$. The class $\mathcal{W}(\alpha, \beta, M)$ contains all differentiable functions $W: \mathbb{R}^{3 \times 3} \rightarrow[0, \infty)$ that satisfy:

(M1) Frame indifference: $W(R F)=W(F)$ for all $F \in \mathbb{R}^{3 \times 3}$ and $R \in \mathrm{SO}(3)$.

(M2) Non-degeneracy and continuity:

$$
\alpha \operatorname{dist}^{2}(F, \mathrm{SO}(3)) \leq W(F) \leq \beta \operatorname{dist}^{2}(F, \mathrm{SO}(3)) \quad \text { for all } F \in \mathbb{R}^{3 \times 3} .
$$

Note that this implies the minimality at the identity, i.e., $W\left(\operatorname{id}_{3 \times 3}\right)=0$.

(M3) Linear stress growth: For the derivative $D W$ of $W$ we have: $|D W(F)| \leq$ $M(|F|+1)$ for all $F \in \mathbb{R}^{3 \times 3}$.

Remark. The condition (M3) is needed here for the first term in the first variation of $\mathcal{E}^{h}$, given in (4), to be well-defined, and thus the condition appears in similar form in [15, 16]. It is however not needed for results concerning $\Gamma$-convergence, e.g., [14]. There also the upper bound (M2) is only needed locally, i.e.,

$$
\exists \rho, \beta^{\prime}>0: W(F) \leq \beta^{\prime} \operatorname{dist}^{2}(F, \operatorname{SO}(3)) \text { for all } F \in \mathbb{R}^{3 \times 3} \text { with } \operatorname{dist}(F, \mathrm{SO}(3)) \leq \rho \text {. }
$$

It is however easily seen that this local upper bound together with linear stress growth implies the global estimate (M2) for some $\beta>0$.

Let now $\alpha, \beta, M$ be as above. A family of energy densities $\left(W^{h}\right)_{h>0}, W^{h}: \Omega \times \mathbb{R}^{3 \times 3} \rightarrow$ $[0, \infty)$ describes an admissible composite material of class $\mathcal{W}(\alpha, \beta, M)$ if for every $h>0$ it holds:

(S1) $W^{h}$ is a Borel function on $\Omega \times \mathbb{R}^{3 \times 3}$.

(S2) $W^{h}(x, \cdot) \in \mathcal{W}(\alpha, \beta, M)$ for almost every $x \in \Omega$.

(S3) There exist a monotone function $r:[0, \infty] \rightarrow[0, \infty]$ and quadratic forms $Q^{h}: \Omega \times \mathbb{R}^{3 \times 3} \rightarrow[0, \infty)$ such that $r(\varepsilon) \searrow 0$ if $\varepsilon \searrow 0$ and

$$
\underset{x \in \Omega}{\operatorname{ess} \sup }\left|W^{h}\left(x, \operatorname{id}_{3 \times 3}+G\right)-Q^{h}(x, G)\right| \leq r(|G|)|G|^{2} \quad \text { for all } G \in \mathbb{R}^{3 \times 3} .
$$

Let $\left(Q^{h}\right)$ be the family of corresponding quadratic forms associated with a family $\left(W^{h}\right) \subset \mathcal{W}(\alpha, \beta, L)$, then it is easy to see that for every $h>0$ we have:

$Q^{h}$ is a Carathéodory function, which for almost every $x \in \Omega$ satisfies

$$
\begin{aligned}
\alpha|\operatorname{sym} F|^{2} \leq Q^{h}(x, F)=Q^{h}(x, \operatorname{sym} F) \leq \beta|\operatorname{sym} F|^{2} & \text { for all } F \in \mathbb{R}^{3 \times 3}, \\
\left|Q^{h}\left(x, F_{1}\right)-Q^{h}\left(x, F_{2}\right)\right| \leq \beta\left|\operatorname{sym} F_{1}-\operatorname{sym} F_{2}\right| & \\
\cdot\left|\operatorname{sym} F_{1}+\operatorname{sym} F_{2}\right| & \text { for all } F_{1}, F_{2} \in \mathbb{R}^{3 \times 3} .
\end{aligned}
$$

Let $\mathbb{A}^{h}$ denote the linear, symmetric, positive semidefinite operator associated with the quadratic forms $Q^{h}$, i.e., $Q^{h}(F)=\frac{1}{2} \mathbb{A}^{h} F: F$ for all $F \in \mathbb{R}^{3 \times 3}$.

In [15, proposition 4.1] the following compactness result was shown: 
Proposition 2.1. Let $\left(u^{h}\right) \subset W^{1,2}\left(\Omega, \mathbb{R}^{3}\right)$ be a sequence satisfying

$$
\limsup _{h \searrow 0} \frac{1}{h^{2}} \int_{\Omega} \operatorname{dist}^{2}\left(\nabla_{h} u^{h}, \mathrm{SO}(3)\right) \mathrm{d} x<\infty .
$$

Then there exists a constant $C>0$, depending only on the domain $\Omega$, and a sequence $\left(R^{h}\right) \subset C^{\infty}([0, L], \mathrm{SO}(3))$, such that

$$
\begin{array}{r}
\left\|\nabla_{h} u^{h}-R^{h}\right\|_{L^{2}(\Omega)} \leq C h \\
\left\|\left(R^{h}\right)^{\prime}\right\|_{L^{2}((0, L))}+h\left\|\left(R^{h}\right)^{\prime \prime}\right\|_{L^{2}((0, L))} \leq C
\end{array}
$$

for every $h>0$. If, in addition, $u^{h}\left(0, x_{2}, x_{3}\right)=\left(0, h x_{2}, h x_{3}\right)$, then

$$
\left|R^{h}(0)-\mathrm{id}\right| \leq C \sqrt{h} .
$$

The following observations are standard, and follow the approach taken in [15. Let $\left(y^{h}\right)$ be the sequence of deformations satisfying the assumptions of Theorem [1.1. The non-degeneracy assumption (M2) implies that $\left(y^{h}\right)$ satisfies (8). Thus by the previous proposition there exists a sequence $\left(R^{h}\right)$ satisfying (9) and (10). By using the frame-indifference of $W^{h}$ we have

$$
\begin{aligned}
W^{h}\left(\cdot, \nabla_{h} y^{h}\right)=W^{h}\left(\cdot,\left(R^{h}\right)^{T} \nabla_{h} y^{h}\right) & =W^{h}\left(\cdot, \operatorname{id}_{3 \times 3}+h \frac{\left(R^{h}\right)^{T} \nabla_{h} y^{h}-\operatorname{id}_{3 \times 3}}{h}\right) \\
& =W^{h}\left(\cdot, \operatorname{id}_{3 \times 3}+h G^{h}\right),
\end{aligned}
$$

where we introduced

$$
G^{h}=\frac{\left(R^{h}\right)^{T} \nabla_{h} y^{h}-\mathrm{id}_{3 \times 3}}{h} .
$$

The estimate (9) implies that $\left(G^{h}\right)$ is uniformly bounded in $L^{2}$. We define $z^{h}$ implicitly by introducing the ansatz

$$
y^{h}(x)=\int_{0}^{x_{1}} R^{h}(s) e_{1} \mathrm{~d} s+h x_{2} R^{h}\left(x_{1}\right) e_{2}+h x_{3} R^{h}\left(x_{1}\right) e_{3}+h z^{h}(x) .
$$

Inserting this ansatz into (12) we can calculate that

$$
G^{h}=\frac{\left(R^{h}\right)^{T} \nabla_{h} y^{h}-\mathrm{id}_{3 \times 3}}{h}=\iota\left(A^{h} \mathfrak{p}\right)+\left(R^{h}\right)^{T} \nabla_{h} z^{h},
$$

where we introduced $A^{h}:=\left(R^{h}\right)^{T}\left(R^{h}\right)^{\prime}$. Clearly $\left(A^{h}\right)$ is uniformly bounded in $L^{2}$, and since $\left(G^{h}\right)$ is uniformly bounded in $L^{2}$ as well, the sequence $\left(\nabla_{h} z^{h}\right)$ is uniformly bounded in $L^{2}$. Furthermore on $\{0\} \times \omega$ we have the boundary conditions $y^{h}(x)=h x_{2} e_{2}+h x_{3} e_{3}$, and thus also we can assume (11) holds. With this we obtain $\left|z^{h}\right| \leq C \sqrt{h}$ on $\{0\} \times \omega$. By applying Poincaré's inequality we can now find a uniform bound on the $L^{2}$-norm of $z^{h}$, and thus on the $W^{1,2}$-norm of $z^{h}$.

Thus, after extracting a subsequence, which we will not relabel, we have in $L^{2}$ the weak convergences

$$
G^{h} \rightarrow G, \quad, \quad A^{h} \rightarrow A \quad \text { and } \quad\left(R^{h}\right)^{T} \nabla_{h} z^{h} \rightarrow R\left(\partial_{1} z\left|q_{2}\right| q_{3}\right)
$$


for some $G \in L^{2}\left(\Omega, \mathbb{R}^{3 \times 3}\right), z \in W^{1,2}\left(\Omega, \mathbb{R}^{3}\right), A \in L^{2}\left((0, L), \mathbb{R}_{\text {skew }}^{3 \times 3}\right)$ and $q_{2}, q_{3} \in$ $L^{2}\left(\Omega, \mathbb{R}^{3}\right)$. Notice that the uniform $L^{2}$ bound on $\left(\frac{1}{h} \partial_{2} z^{h}, \frac{1}{h} \partial_{3} z^{h}\right)$ implies that $z$ does not depend on $x_{2}, x_{3}$. Thus going to the limit in (14) we obtain

$$
G(x)=\iota\left(p\left(x_{1}\right)+A\left(x_{1}\right) \mathfrak{p}(x)\right)+R\left(x_{1}\right)^{T}\left(0\left|q_{2}(x)\right| q_{3}(x)\right),
$$

where for brevity we set $p:=R^{T} \partial_{1} z \in L^{2}\left((0, L), \mathbb{R}^{3}\right)$. Next we focus on sym $G^{h}$. In 14, proof of theorem 2.15] it is shown that there exist sequences $v^{h} \subset W^{1,2}\left(\Omega, \mathbb{R}^{3}\right)$, $\left(\Psi^{h}\right) \subset W^{1,2}\left((0, L), \mathbb{R}_{\text {skew }}^{3 \times 3}\right)$ and $o^{h} \subset L^{2}\left(\Omega, \mathbb{R}^{3 \times 3}\right)$ such that

$$
\operatorname{sym} G^{h}=\operatorname{sym} \iota\left(A \mathfrak{p}+p_{1} e_{1}\right)+\operatorname{sym} \iota\left(\left(\Psi^{h}\right)^{\prime} \mathfrak{p}\right)+\operatorname{sym} \nabla_{h} v^{h}+o^{h},
$$

and such that $\left(\nabla_{h} v^{h}\right)$ is uniformly bounded in $L^{2}$, and $\Psi^{h} \rightarrow 0$ in $W^{1,2}\left((0, L), \mathbb{R}^{3 \times 3}\right)$, $v^{h} \rightarrow 0$ in $W^{1,2}\left(\Omega, \mathbb{R}^{3}\right)$ and $o^{h} \rightarrow 0$ strongly in $L^{2}\left(\Omega, \mathbb{R}^{3 \times 3}\right)$.

We define the fixed part $m_{d}$ by

$$
m_{d}:=A \mathfrak{p}+p_{1} e_{1}
$$

and the corrector sequence $\left(\psi^{h}\right)$ by

$$
\psi^{h}(x)=\Psi^{h} \mathfrak{p}-\frac{1}{h}\left(\widehat{\Psi}_{12}^{h} e_{2}+\widehat{\Psi}_{13}^{h} e_{3}\right)+v^{h},
$$

where $\widehat{\Psi}^{h}\left(x_{1}\right)=\int_{0}^{x_{1}} \Psi^{h}(s) \mathrm{d} s$. Direct calculation yields

$$
\nabla_{h} \psi^{h}=\left(\left(\Psi^{h}\right)^{\prime} \mathfrak{p}-\frac{1}{h} \Psi_{12}^{h} e_{2}-\frac{1}{h} \Psi_{13}^{h} e_{3}, \quad \frac{1}{h} \Psi^{h} e_{2}, \quad \frac{1}{h} \Psi^{h} e_{3}\right)+\nabla_{h} v^{h},
$$

as well as

$$
\operatorname{sym} \nabla_{h} \psi^{h}=\operatorname{sym} \iota\left(\left(\Psi^{h}\right)^{\prime} \mathfrak{p}\right)+\operatorname{sym} \nabla_{h} v^{h} .
$$

Thus we have

$$
\operatorname{sym} G^{h}=\operatorname{sym} \iota\left(m_{d}\right)+\operatorname{sym} \nabla_{h} \psi^{h}+o^{h},
$$

with easily verifiable strong convergences

$$
\left(\psi_{1}^{h}, h \psi_{2}^{h}, h \psi_{3}^{h}\right) \rightarrow 0 \text { in } L^{2}\left(\Omega, \mathbb{R}^{3}\right) \quad \text { and } \quad \mathrm{t}\left(\psi_{2}^{h}, \psi_{3}^{h}\right) \rightarrow 0 \text { in } L^{2}((0, L)) .
$$

\subsection{The $\Gamma$-limit}

We will briefly introduce the variational approach developed in [14], with which the $\Gamma$-convergence for the inhomogeneous rod was proved. A similar variational approach for thin elastica was used earlier in 4 for the membrane model. The approach was also already adapted and used in [5] to show the convergence of stationary points for the inhomogeneous von Kármán rod.

By applying the frame indifference (M1) and Taylor expansion (S3) we obtain

$$
\begin{aligned}
\frac{1}{h^{2}} W^{h}\left(\cdot, \nabla_{h} y^{h}\right) & =\frac{1}{h^{2}} W^{h}\left(\cdot, \operatorname{id}_{3 \times 3}+h G^{h}\right) \\
& \approx \frac{1}{h^{2}} Q^{h}\left(\cdot, h G^{h}\right)=Q^{h}\left(\cdot, G^{h}\right)=Q^{h}\left(\cdot, \operatorname{sym} G^{h}\right) .
\end{aligned}
$$


This motivates, together with the decomposition (18), the definitions

$$
\begin{aligned}
\mathcal{K}_{(h)}^{-}(m, O) & :=\inf \left\{\liminf _{h \searrow 0} \int_{O \times \omega} Q^{h}\left(x, \iota(m)+\nabla_{h} \psi^{h}\right) \mathrm{d} x\right\}, \\
\mathcal{K}_{(h)}^{+}(m, O) & :=\inf \left\{\limsup _{h \searrow 0} \int_{O \times \omega} Q^{h}\left(x, \iota(m)+\nabla_{h} \psi^{h}\right) \mathrm{d} x\right\},
\end{aligned}
$$

where we take the infimum over all sequences $\left(\psi^{h}\right) \subset W^{1,2}\left(O \times \omega, \mathbb{R}^{3}\right)$ such that $\left(\psi_{1}^{h}, h \psi_{2}^{h}, h \psi_{3}^{h}\right) \rightarrow 0$ strongly in $L^{2}\left(O \times \omega, \mathbb{R}^{3}\right) \quad$ and $\quad \mathrm{t}\left(\psi_{2}^{h}, \psi_{3}^{h}\right) \rightarrow 0 \quad$ in $L^{2}(O)$.

It is proved in [14, lemma 2.6] that there exists a subsequence, still denoted by $(h)$, such that:

$$
\forall m \in L^{2}\left(\Omega, \mathbb{R}^{3}\right), \forall O \subset[0, L] \text { open }: \mathcal{K}_{(h)}(m, O):=\mathcal{K}_{(h)}^{-}(m, O)=\mathcal{K}_{(h)}^{+}(m, O) .
$$

This can be done by extracting a diagonal sequence such that $\mathcal{K}_{(h)}^{-}$and $\mathcal{K}_{(h)}^{+}$agree on a dense, countable subset of $L^{2}$ and of open subsets of $(0, L)$. Utilizing the continuity of the maps $L^{2}\left(\Omega, \mathbb{R}^{3}\right) \rightarrow \mathbb{R}, m \mapsto \mathcal{K}_{(h)}^{-}(m, O), m \mapsto \mathcal{K}_{(h)}^{+}(m, O)$ for any open set $O \subset(0, L)$, proved in [14, lemma 2.5], it is then easy to see that (20) holds. We now introduce the relaxation sequence and state its most important properties, which were proved in [5] and [14]:

Lemma 2.2. Let $(h) \subset(0, \infty)$ with $h \searrow 0$ be a sequence such that (20) holds true. Then there exists a subsequence (not relabeled) such that for every $m \in L^{2}\left(\Omega, \mathbb{R}^{2}\right.$ ) there exists $\left(\psi_{m}^{h}\right) \subset W^{1,2}\left(\Omega, \mathbb{R}^{2}\right)$, with the property that for every open set $O \subset(0, L)$ we have

$$
\mathcal{K}_{(h)}(m, O):=\lim _{h \searrow 0} \int_{O \times \omega} Q^{h}\left(x, \iota(m)+\nabla_{h} \psi_{m}^{h}\right) \mathrm{d} x,
$$

and $\left(\psi_{m}^{h}\right)$ satisfies the following properties:

(a) $\left(\psi_{m}^{h} \cdot e_{1}, h \psi_{m}^{h} \cdot e_{2}, h \psi_{m}^{h} \cdot e_{3}\right) \rightarrow 0$ and $\mathrm{t}\left(\psi_{m}^{h} \cdot e_{2}, \psi_{m}^{h} \cdot e_{3}\right) \rightarrow 0$ strongly in $L^{2}$.

(b) The sequence $\left(\left|\operatorname{sym} \nabla \psi_{m}^{h}\right|^{2}\right)$ is equi-integrable, and there exist sequences $B_{m}^{h} \subset$ $W^{1,2}\left((0, L), \mathbb{R}_{\text {skew }}^{3 \times 3}\right)$ and $\left(\vartheta_{m}^{h}\right) \subset W^{1,2}\left(\Omega, \mathbb{R}^{3}\right)$ with $B_{m}^{h} \rightarrow 0, \vartheta_{m}^{h} \rightarrow 0$ strongly in their respective $L^{2}$-norm, and

$$
\operatorname{sym} \nabla_{h} \psi_{m}^{h}=\operatorname{sym} \iota\left(\left(B_{m}^{h}\right)^{\prime} \mathfrak{p}\right)+\operatorname{sym} \nabla_{h} \vartheta_{m}^{h} .
$$

Moreover, for a subsequence $\left(\left|\left(B_{m}^{h}\right)^{\prime}\right|^{2}\right)$ and $\left(\left|\nabla_{h} \vartheta_{m}^{h}\right|^{2}\right)$ are equi-integrable, and the following inequality holds for some $C>0$ independent of $O \subset(0, L)$ :

$$
\limsup _{h \searrow 0}\left(\left\|B_{m}^{h}\right\|_{W^{1,2}(O)}+\left\|\nabla_{h} \vartheta_{m}^{h}\right\|_{L^{2}(O \times \omega)}\right) \leq C\left(\beta\|m\|_{L^{2}(O \times \omega)}^{2}+1\right) .
$$

(c) If $\left(\widehat{\psi}^{h}\right) \subset W^{1,2}\left(\Omega, \mathbb{R}^{3}\right)$ is any other sequence that satisfies (a) and $\left(\operatorname{sym} \nabla_{h} \widehat{\psi}^{h}\right)$ is bounded in $L^{2}\left(\Omega, \mathbb{R}^{3 \times 3}\right)$, then

$$
\lim _{h \searrow 0} \int_{\Omega} \mathbb{A}^{h}\left(\iota(m)+\nabla_{h} \psi_{m}^{h}\right): \operatorname{sym} \nabla_{h} \widehat{\psi}^{h} \mathrm{~d} x=0 .
$$


(d) If $\left(\widehat{\psi}^{h}\right) \subset W^{1,2}\left(\Omega, \mathbb{R}^{3}\right)$ is any sequence that satisfies (21) and (a), then

$$
\left\|\operatorname{sym} \nabla_{h} \psi_{m}^{h}-\operatorname{sym} \nabla_{h} \widehat{\psi}^{h}\right\|_{L^{2}(\Omega)} \rightarrow 0,
$$

and $\left(\left|\operatorname{sym} \nabla_{h} \widehat{\psi}^{h}\right|^{2}\right)$ is equi-integrable.

(e) The map $\mathcal{K}_{(h)}(\cdot,(0, L)): L^{2}\left(\Omega, \mathbb{R}^{3}\right) \rightarrow \mathbb{R}$ is continuously Fréchet-differentiable, and for every $m, n \in L^{2}\left(\Omega, \mathbb{R}^{3}\right)$ we have

$$
\frac{\partial \mathcal{K}_{(h)}(m,(0, L))}{\partial m}[n]=\lim _{h \searrow 0} \int_{\Omega} \mathbb{A}^{h}\left(\iota(m)+\operatorname{sym} \nabla_{h} \psi_{m}^{h}\right): \iota(n) \mathrm{d} x .
$$

The sequence $\left(\psi_{m}^{h}\right)$ is called the relaxation sequence for $m$. For our purposes $m$ will always be of the form $m=B \mathfrak{p}+b e_{1}$ for some $B \in L^{2}\left((0, L), \mathbb{R}_{\text {skew }}^{3 \times 3}\right)$ and $b \in$ $L^{2}((0, L))$. Thus we introduce the linear map

$$
m: L^{2}\left((0, L), \mathbb{R}_{\text {skew }}^{3 \times 3}\right) \times L^{2}((0, L), \mathbb{R}) \rightarrow L^{2}\left(\Omega, \mathbb{R}^{3}\right), \quad m(B, b):=B \mathfrak{p}+b e_{1} .
$$

By applying the chain rule together with $(e)$ we thus can deduce the derivative of $\mathcal{K}_{(h)}(\cdot,(0, L)) \circ m$. Indeed, for every $B, M \in W^{1,2}\left((0, L), \mathbb{R}_{\text {skew }}^{3 \times 3}\right), b, \mu \in L^{2}((0, L))$ we have

$$
\begin{aligned}
\frac{\partial \mathcal{K}_{(h)}(m(B, b),(0, L))}{\partial B}[M] & =\lim _{h \searrow 0} \int_{\Omega} \mathbb{A}^{h}\left(\iota(m(B, b))+\operatorname{sym} \nabla_{h} \psi_{m(B, b)}^{h}\right): \iota(M \mathfrak{p}) \mathrm{d} x, \\
\frac{\partial \mathcal{K}_{(h)}(m(B, b),(0, L))}{\partial b}[\mu] & =\lim _{h \searrow 0} \int_{\Omega} \mathbb{A}^{h}\left(\iota(m(B, b))+\operatorname{sym} \nabla_{h} \psi_{m(B, b)}^{h}\right): \iota\left(\mu e_{1}\right) \mathrm{d} x .
\end{aligned}
$$

To shorten notation we define $\mathcal{K}(m):=\mathcal{K}(m,(0, L))$ for every $m \in L^{2}$.

In [14, proposition 2.12] also the following result regarding the existence of a density for $\mathcal{K}_{(h)}$ was proved:

Proposition 2.3. Let $(h) \subset(0, \infty)$ with $h \searrow 0$ be a sequence such that (20) holds true for every $m \in L^{2}\left(\Omega, \mathbb{R}^{3}\right)$. Then a measurable function $Q^{0}:[0, L] \times$ $\mathbb{R}_{\text {skew }}^{3 \times 3} \times \mathbb{R} \rightarrow[0, \infty)$ exists, such that for every $O \subset[0, L]$ open, and every $(B, b) \in$ $L^{2}\left((0, L), \mathbb{R}_{\text {skew }}^{3 \times 3} \times \mathbb{R}\right)$ we have

$$
\mathcal{K}_{(h)}(m(B, b), O)=\int_{O} Q^{0}\left(x_{1}, B\left(x_{1}\right), b\left(x_{1}\right)\right) \mathrm{d} x_{1} .
$$

Furthermore for almost every $x_{1} \in[0, L]$ the map $Q^{0}\left(x_{1}, \cdot, \cdot\right)$ is a quadratic form, and there exists $C=C(\omega)>0$ independent of $x_{1}$, such that for every $\widehat{B} \in \mathbb{R}_{\text {skew }}^{3 \times 3}, \widehat{b} \in \mathbb{R}$ we have

$$
C^{-1}\left(|\widehat{B}|^{2}+|\widehat{b}|^{2}\right) \leq Q^{0}\left(x_{1}, \widehat{B}, \widehat{b}\right) \leq C \beta\left(|\widehat{B}|^{2}+|\widehat{b}|^{2}\right) .
$$

From this we easily deduce that the map $\widehat{b}_{\text {min }}:[0, L] \times \mathbb{R}_{\text {skew }}^{3 \times 3} \rightarrow \mathbb{R}$, given by $\widehat{b}_{\min }\left(x_{1}, \widehat{B}\right)=\arg \min _{b \in \mathbb{R}} Q^{0}\left(x_{1}, \widehat{B}, b\right)$ is well-defined, linear in $\widehat{B}$, and there exists a constant $C^{\prime}=C^{\prime}(\alpha, \beta, \omega)>0$ such that for almost every $x_{1}$ and all $\widehat{B} \in \mathbb{R}_{\text {skew }}^{3 \times 3}$ we have

$$
\left|\widehat{b}_{\min }\left(x_{1}, \widehat{B}\right)\right| \leq C^{\prime}|\widehat{B}| .
$$


Finally we can define the density for the limiting bending energy. Let map $Q_{1}^{0}$ : $[0, L] \times \mathbb{R}_{\text {skew }}^{3 \times 3} \rightarrow \mathbb{R}$ be given by $Q_{1}^{0}\left(x_{1}, \widehat{B}\right):=Q^{0}\left(x_{1}, \widehat{B}, \widehat{b}_{\text {min }}\left(x_{1}, \widehat{B}\right)\right)$. It is easily seen that:

For almost every $x_{1} \in[0, L]$ the map $Q_{1}^{0}\left(x_{1}, \cdot\right)$ is a quadratic form, and there exists $C^{\prime \prime}=C^{\prime \prime}(\alpha, \beta, \omega)>0$ such that for all $\hat{B} \in \mathbb{R}_{\text {skew }}^{3 \times 3}$ it holds

$$
\left(C^{\prime \prime}\right)^{-1}|\widehat{B}|^{2} \leq Q_{1}^{0}\left(x_{1}, \widehat{B}\right) \leq C^{\prime \prime}|\widehat{B}|^{2} .
$$

We now define the limiting bending energy $\mathcal{K}_{(h)}^{0}: L^{2}\left((0, L), \mathbb{R}_{\text {skew }}^{3 \times 3}\right) \rightarrow \mathbb{R}$ simply by integrating over the density $Q_{1}^{0}$, i.e.,

$$
\begin{aligned}
\mathcal{K}_{(h)}^{0}(B) & :=\int_{0}^{L} Q_{1}^{0}\left(x_{1}, B\left(x_{1}\right)\right) \mathrm{d} x_{1} \\
& =\int_{0}^{L} Q^{0}\left(x_{1}, B\left(x_{1}\right), \widehat{b}_{\min }\left(x_{1}, B\left(x_{1}\right)\right)\right) \mathrm{d} x_{1} .
\end{aligned}
$$

From the linearity of $\widehat{B} \mapsto \widehat{b}_{\min }(\cdot, \widehat{B})$ and the Fréchet-differentiability of $\mathcal{K}_{(h)}$ we deduce that also $\mathcal{K}_{(h)}^{0}$ is Fréchet-differentiable. For fixed $\widehat{B} \in \mathbb{R}_{\text {skew }}^{3 \times 3}$ and almost every $x_{1}$ the function $Q^{0}\left(x_{1}, \widehat{B}, \cdot\right)$ has quadratic growth, thus $\widehat{b}_{\min }\left(x_{1}, \widehat{B}\right)$ is the unique stationary point of $Q^{0}\left(x_{1}, \widehat{B}, \cdot\right)$, i.e.,

$$
\left(\partial_{b} Q^{0}\right)\left(x_{1}, \widehat{B}, b\right)=0 \Longleftrightarrow b=\widehat{b}_{\min }\left(x_{1}, \widehat{B}\right) .
$$

Furthermore the mapping $b_{\text {min }}: L^{2}\left((0, L), \mathbb{R}_{\text {skew }}^{3 \times 3}\right) \rightarrow L^{2}((0, L)), b_{\min }(B)=\widehat{b}_{\min }(\cdot, B)$ is linear and well-defined. Thus for any $B \in L^{2}\left((0, L), \mathbb{R}_{\text {skew }}^{3 \times 3}\right)$ and $b \in L^{2}((0, L))$ we have

$$
\left\{\frac{\partial \mathcal{K}_{(h)}(m(B, b))}{\partial b}[\mu]=0 \quad \text { for all } \mu \in L^{2}((0, L))\right\} \Longleftrightarrow b=b_{\min }(B) .
$$

We are now able to compute the variations of $\mathcal{K}_{(h)}^{0}$. For fixed $B, M \in L^{2}\left((0, L), \mathbb{R}_{\text {skew }}^{3 \times 3}\right)$ we calculate by using the chain rule

$$
\begin{aligned}
\left(\frac{\partial}{\partial B} \mathcal{K}_{(h)}^{0}\right)(B)[M] & =\frac{\partial}{\partial B}\left(\mathcal{K}_{(h)}\left(m\left(B, b_{\min }(B)\right)\right)\right)[M] \\
& =\left(\frac{\partial \mathcal{K}_{(h)}}{\partial m}\left(m\left(B, b_{\min }(B)\right)\right)\right)\left[\frac{\partial m\left(B, b_{\min }(B)\right)}{\partial B}[M]\right] .
\end{aligned}
$$

From (23) and the linearity of $b_{\min }$ we obtain

$$
\frac{\partial m\left(B, b_{\min }(B)\right)}{\partial B}[M]=M \mathfrak{p}+\left(\left(\partial_{B} b_{\min }\right)(0): M\right) e_{1}
$$

and thus (26) can be rewritten to

$$
\begin{aligned}
\left(\frac{\partial}{\partial B} \mathcal{K}_{(h)}^{0}\right)(B)[M]=\lim _{h \searrow 0} \int_{\Omega} \mathbb{A}^{h}\left(\iota\left(m\left(B, b_{\min }(B)\right)\right)\right. & +\operatorname{sym} \nabla_{h} \psi_{\left.m\left(B, b_{\min }(B)\right)\right)}^{h} \\
& : \iota\left(M \mathfrak{p}+\left(\left(\partial_{B} b_{\min }\right)(0): M\right) e_{1}\right) .
\end{aligned}
$$


The function $b_{\min }(B)$ satisfies according to (25) the equation

$$
\begin{aligned}
0 & =\frac{\partial \mathcal{K}_{(h)}}{\partial b}\left(m\left(B, b_{\min }(B)\right)[\mu]\right. \\
& =\lim _{h \searrow 0} \int_{\Omega} \mathbb{A}^{h}\left(\iota\left(m\left(B, b_{\min }(B)\right)\right)+\operatorname{sym} \nabla_{h} \psi_{m\left(B, b_{\min }(B)\right)}^{h}\right): \iota\left(\mu e_{1}\right)
\end{aligned}
$$

for all $\mu \in L^{2}((0, L))$. Finally using $\mu:=\left(\partial_{B} b_{\min }\right)(0): M$ in (28) allows us to simplify (27) to

$$
\left(\frac{\partial}{\partial B} \mathcal{K}_{(h)}^{0}\right)(B)[M]=\lim _{h \searrow 0} \int_{\Omega} \mathbb{A}^{h}\left(\iota\left(m\left(B, b_{\min }(B)\right)\right)+\operatorname{sym} \nabla_{h} \psi_{m\left(B, b_{\min }(B)\right)}^{h}\right): \iota(M \mathfrak{p}) .
$$

\subsection{Derivation of the limit Euler-Lagrange equation}

Let $\left(y, d_{2}, d_{3}\right) \in \mathcal{A}$, where $\mathcal{A}$ is give in (3), and assume, in addition, that $y(0)=0$. The associated rotation function is then given by $R=\left(y^{\prime}, d_{2}, d_{3}\right) \in W^{1,2}((0, L), \mathrm{SO}(3))$. Recall that the limit energy of $\left(y, d_{2}, d_{3}\right)$ is

$$
\begin{aligned}
\mathcal{E}^{0}\left(y, d_{2}, d_{3}\right) & =\mathcal{K}_{(h)}^{0}\left(R^{T} R^{\prime}\right)-\int_{0}^{L} g \cdot y \mathrm{~d} x_{1} \\
& =\mathcal{K}_{(h)}^{0}\left(R^{T} R^{\prime}\right)-\int_{0}^{L} \widehat{g} \cdot y^{\prime} \mathrm{d} x_{1},
\end{aligned}
$$

with $\widehat{g}\left(x_{1}\right)=\int_{x_{1}}^{L} g(s) \mathrm{d} s$. We say $\left(y, d_{2}, d_{3}\right)$ is a stationary point of $\mathcal{E}^{0}$, if for any $C^{1}$-curve $\gamma:(-\infty, \infty) \rightarrow \mathcal{A}$ with $\gamma(0)=\left(y, d_{2}, d_{3}\right)$ we have

$$
\left.\left(\partial_{\varepsilon} \mathcal{E}^{0}[\gamma(\varepsilon)]\right)\right|_{\varepsilon=0}=D \mathcal{E}^{0}\left[y, d_{2}, d_{3}\right][\dot{\gamma}(0)]=0,
$$

where $\dot{\gamma}$ denotes the derivative of $\gamma$. The following lemma gives an alternative characterization by identifying the tangent spaces of $\mathcal{A}$ and explicitly computing the derivative $D \mathcal{E}^{0}$.

Lemma 2.4. Let $\left(y, d_{2}, d_{3}\right) \in \mathcal{A}$. Define $R=\left(y^{\prime}, d_{2}, d_{3}\right)$ and $A=R^{T} R^{\prime}$. Then $\left(y, d_{2}, d_{3}\right)$ is a stationary point of $\mathcal{E}^{0}$ iff. for every $\Phi \in W^{1,2}\left((0, L), \mathbb{R}_{\text {skew }}^{3 \times 3}\right)$ we have

$$
\left(\frac{\partial}{\partial B} \mathcal{K}_{(h)}^{0}\right)(A)\left[A \Phi-\Phi A+\Phi^{\prime}\right]=\int_{0}^{L} \widehat{g} \cdot\left(R \Phi e_{1}\right) \mathrm{d} x_{1} .
$$

Proof. Let $\left(y^{\varepsilon}, d_{2}^{\varepsilon}, d_{3}^{\varepsilon}\right)_{\varepsilon} \subset \mathcal{A}$ be a $C^{1}$-curve with $\left(y^{0}, d_{2}^{0}, d_{3}^{0}\right)=\left(y, d_{2}, d_{3}\right)$, and define $\left(R_{\varepsilon}\right)_{\varepsilon} \subset W^{1,2}((0, L), \mathrm{SO}(3))$ by $R_{\varepsilon}=\left(\left(y^{\varepsilon}\right)^{\prime}, d_{2}^{\varepsilon}, d_{3}^{\varepsilon}\right)$; especially we have $R_{0}=R$. It is well-known that the tangent space of $\mathrm{SO}(3)$ in $R$ is given by $\left\{R \Phi: \Phi \in \mathbb{R}_{\text {skew }}^{3 \times 3}\right\}$. Thus, denoting the derivative of $\left(R_{\varepsilon}\right)$ with respect to $\varepsilon$ by $\left(\dot{R}_{\varepsilon}\right)$, we obtain $R_{0}^{T} \dot{R}_{0}=\Phi$ for some $\Phi \in W^{1,2}\left((0, L), \mathbb{R}_{\text {skew }}^{3 \times 3}\right)$ and the tangent space of $\mathcal{A}$ in $\left(y, d_{2}, d_{3}\right)$ is given by

$$
\begin{aligned}
T_{\left(y, d_{2}, d_{3}\right)} \mathcal{A}=\left\{\left(v_{1}, v_{2}, v_{3}\right) \in W^{2,2}\left((0, L), \mathbb{R}^{3}\right) \times W^{1,2}\left((0, L), \mathbb{R}^{3}\right)^{2}:\right. & \\
\left(y^{\prime}, d_{2}, d_{3}\right)^{T}\left(v_{1}^{\prime}, v_{2}, v_{3}\right) & \left.\in W^{1,2}\left((0, L), \mathbb{R}_{\text {skew }}^{3 \times 3}\right)\right\} .
\end{aligned}
$$


With the chain rule we obtain

$$
\begin{aligned}
\left.\partial_{\varepsilon} \mathcal{E}^{0}\left[y^{\varepsilon}, d_{2}^{\varepsilon}, d_{3}^{\varepsilon}\right]\right|_{\varepsilon=0} & =\left.\partial_{\varepsilon}\left(\mathcal{K}_{(h)}^{0}\left(R_{\varepsilon}^{T} R_{\varepsilon}^{\prime}\right)-\int_{0}^{L} \widehat{g} \cdot y_{\varepsilon}^{\prime}\right)\right|_{\varepsilon=0} \\
& =\left.\frac{\partial \mathcal{K}_{(h)}^{0}}{\partial B}\left(R^{T} R^{\prime}\right)\left[\partial_{\varepsilon}\left(R_{\varepsilon}^{T} R_{\varepsilon}^{\prime}\right)\right]\right|_{\varepsilon=0}-\int_{0}^{L} \widehat{g} \cdot\left(R_{0} \Phi e_{1}\right) \\
& =\frac{\partial \mathcal{K}_{(h)}^{0}}{\partial B}\left(R^{T} R^{\prime}\right)\left[\dot{R}_{0}^{T} R_{0}^{\prime}+R_{0}^{T} \dot{R}_{0}^{\prime}\right]-\int_{0}^{L} \widehat{g} \cdot\left(R_{0} \Phi e_{1}\right) .
\end{aligned}
$$

By using the relationship $R_{0}^{T} \dot{R}_{0}=\Phi$ we obtain

$$
\dot{R}_{0}^{T} R_{0}^{\prime}=-\Phi R^{T} R^{\prime} \quad \text { and } \quad R_{0}^{T} \dot{R}_{0}^{\prime}=R^{T}(R \Phi)^{\prime}=R^{T} R^{\prime} \Phi+R^{T} R \Phi^{\prime},
$$

and thus

$$
\dot{R}_{0}^{T} R_{0}^{\prime}+R_{0}^{T} \dot{R}_{0}^{\prime}=-\Phi R^{T} R^{\prime}+R^{T} R^{\prime} \Phi+\Phi^{\prime} .
$$

Furthermore we can insert $A=R^{T} R^{\prime}$ into the equality, which finally reads

$$
\dot{R}_{0}^{T} R_{0}^{\prime}+R_{0}^{T} \dot{R}_{0}^{\prime}=-\Phi A+A \Phi+\Phi^{\prime} .
$$

Defining

$$
x_{1} \mapsto \widehat{\Phi}\left(x_{1}\right)=\left(\int_{0}^{x_{1}} R(s) \Phi(s) e_{1} \mathrm{~d} s,(R \Phi)\left(x_{1}\right) e_{2},(R \Phi)\left(x_{1}\right) e_{3}\right) \in T_{\left(y, d_{2}, d_{3}\right)} \mathcal{A}
$$

we thus get

$$
\left(D \mathcal{E}^{0}\right)\left(y, d_{2}, d_{3}\right)[\widehat{\Phi}]=\frac{\partial \mathcal{K}_{(h)}^{0}}{\partial B}(A)\left[A \Phi-\Phi A+\Phi^{\prime}\right]-\int_{0}^{L} \widehat{g} \cdot\left(R \Phi e_{1}\right) .
$$

If $\left(y, d_{2}, d_{3}\right)$ is stationary, we left-hand side vanishes and we obtain as claimed

$$
\frac{\partial \mathcal{K}_{(h)}^{0}}{\partial B}(A)\left[A \Phi-\Phi A+\Phi^{\prime}\right]=\int_{0}^{L} \widehat{g} \cdot\left(R \Phi e_{1}\right) .
$$

\section{Proof of the main theorem}

We dedicate the whole section to the proof of Theorem 1.1. From now on let $W^{h}, y^{h}, g$ be as in Theorem [1.1. From the energy bound (5) together with the non-degeneracy hypothesis (M2) on $W^{h}$ we obtain the inequality

$$
\limsup _{h \searrow 0}\left\|\operatorname{dist}\left(\nabla_{h} y^{h}, \mathrm{SO}(3)\right)\right\|_{L^{2}}<\infty,
$$

and furthermore by assumption on $\left(y^{h}\right)$ we have that $y^{h}\left(0, x_{2}, x_{3}\right)=h x_{2} e_{2}+h x_{3} e_{3}$. Thus we may apply Proposition 2.1 and deduce that there exists a sequence of rotations $\left(R^{h}\right) \subset C^{\infty}([0, L], \mathrm{SO}(3))$ with properties (9), (10) and (11). 
We recall the definition of the linearized strain $G^{h}$ given by

$$
G^{h}=\frac{\left(R^{h}\right)^{T} \nabla_{h} y^{h}-\mathrm{id}_{3 \times 3}}{h} .
$$

It was already introduced in (12) and, by the discussion following the definition, there exist a subsequence (not relabeled) and a function $G \in L^{2}\left(\Omega, \mathbb{R}^{3 \times 3}\right.$ ) such that $G^{h} \rightarrow G$ in $L^{2}$. From the frame indifference of $W^{h}$ it follows that

$$
D W^{h}(x, F)=R D W^{h}\left(x, R^{T} F\right) \quad \text { for all } F \in \mathbb{R}^{3 \times 3}, R \in \mathrm{SO}(3) \text {, a.e. } x \in \Omega \text {. }
$$

Thus

$$
D W^{h}\left(x, \nabla_{h} y^{h}\right)=R^{h} D W^{h}\left(x, \operatorname{id}_{3 \times 3}+h G^{h}\right)=h R^{h} E^{h},
$$

where $E^{h}:=h^{-1} D W^{h}\left(\cdot, \operatorname{id}_{3 \times 3}+h G^{h}\right)$ is the nonlinear stress. On the other hand a Taylor expansion around the identity yields

$$
D W^{h}\left(x, \operatorname{id}_{3 \times 3}+h G^{h}\right)=h D^{2} W^{h}\left(x, \operatorname{id}_{3 \times 3}\right) G^{h}+\zeta^{h}\left(x, h G^{h}\right) .
$$

where (S3) implies the estimate

$$
\left|\zeta^{h}(x, F)\right| \leq \widehat{r}(|F|)|F|,
$$

for some monotone $\widehat{r}:[0, \infty) \rightarrow[0, \infty)$ with $\widehat{r}(\varepsilon) \searrow 0$ if $\varepsilon \searrow 0$. Together with $D^{2} W^{h}\left(\cdot, \operatorname{id}_{3 \times 3}\right)=D^{2} Q^{h}(\cdot, 0)=\mathbb{A}^{h}$ we get

$$
E^{h}=\mathbb{A}^{h} \operatorname{sym} G^{h}+\frac{1}{h} \zeta^{h}\left(\cdot, h G^{h}\right) .
$$

The error term $h^{-1} \zeta^{h}\left(\cdot, h G^{h}\right)$ does not necessarily converge strongly to 0 in $L^{2}$, since $G^{h}$ might concentrate in $L^{2}$. We will now show that the error term does not oscillates, and that it weakly converges to zero:

Lemma 3.1. Let $\left(\eta^{h}\right) \subset L^{2}(\Omega)$ be such that $\left(\left|\eta^{h}\right|^{2}\right)$ is equi-integrable. Then

$$
\lim _{h \searrow 0} \int_{\Omega} \eta^{h} \cdot\left(\frac{1}{h} \zeta^{h}\left(\cdot, h G^{h}\right)\right) \mathrm{d} x=0 .
$$

Note that this immediately implies $h^{-1} \zeta^{h}\left(\cdot, h G^{h}\right) \rightarrow 0$ in $L^{2}\left(\Omega, \mathbb{R}^{3 \times 3}\right)$, and especially that $\left(h^{-1} \zeta^{h}\left(\cdot, h G^{h}\right)\right)$ is uniformly bounded in $L^{2}$.

Proof. Let $0<\alpha<1$. We define the sets $S_{h}^{\alpha}=\left\{x \in \Omega: \quad h\left|G^{h}(x)\right| \leq h^{\alpha}\right\}$, and the truncated function $\widehat{G}^{h}:=G^{h} \chi_{S_{h}^{\alpha}}$. Obviously $h \widehat{G}^{h} \rightarrow 0$ in $L^{\infty}, G^{h}=\widehat{G}^{h}$ on $S_{h}^{\alpha}$, and by Chebyshev inequality we have $\mathcal{L}^{2}\left(\Omega \backslash S_{h}^{\alpha}\right) \rightarrow 0$ for $h \searrow 0$. We can now compute

$$
\begin{aligned}
\left\|\frac{1}{h} \zeta^{h}\left(\cdot, h \widehat{G}^{h}\right)\right\|_{L^{2}}^{2} & =\frac{1}{h^{2}} \int_{\Omega}\left|\zeta^{h}\left(x, h \widehat{G}^{h}\right)\right|^{2} \mathrm{~d} x \\
& \leq \frac{1}{h^{2}} \int_{\Omega} \widehat{r}\left(\left\|h \widehat{G}^{h}\right\|_{\infty}\right)^{2}\left|h \widehat{G}^{h}\right|^{2} \mathrm{~d} x \\
& \leq \widehat{r}\left(\left\|h \widehat{G}^{h}\right\|_{\infty}\right)^{2}\left\|\widehat{G}^{h}\right\|_{L^{2}}^{2} \leq \widehat{r}\left(\left\|h \widehat{G}^{h}\right\|_{\infty}\right)^{2}\left\|G^{h}\right\|_{L^{2}}^{2} \rightarrow 0,
\end{aligned}
$$


by the uniform bound of $G^{h}$ in the $L^{2}$-norm. Finally applying Hölder's inequality yields

$$
\left|\int_{\Omega} \eta^{h} \cdot\left(\frac{1}{h}\left(\zeta^{h}\left(x, h G^{h}\right)-\zeta^{h}\left(x, h \widehat{G}^{h}\right)\right)\right) \mathrm{d} x\right| \leq C \int_{S_{h}}\left|\eta^{h}\right|^{2} \mathrm{~d} x \rightarrow 0,
$$

which implies the claim.

With this result we can deduce the limit PDE in terms of the stress. The part follows closely the corresponding proof in [15], and thus we skip some details.

\section{Compactness}

From the properties (9)-(11) for the sequence $\left(R^{h}\right)$, we deduce that there exist a subsequence (not relabeled) and limit $R \subset W^{1,2}((0, L), \mathrm{SO}(3))$ such that $R(0)=$ $\operatorname{id}_{3 \times 3}$ and $R^{h} \rightarrow R$ in $W^{1,2}((0, L), \mathrm{SO}(3))$. Defining $\bar{y}\left(x_{1}\right)=\int_{0}^{x_{1}} R(s) e_{1} \mathrm{~d} s, \bar{d}_{k}=R e_{k}$ for $k=2,3$ we obtain $\bar{y} \in W_{\text {bdy }}^{2,2}\left([0, L], \mathbb{R}^{3}\right), y^{h} \rightarrow \bar{y}$ strongly in $W^{1,2}\left(\Omega, \mathbb{R}^{3}\right), \nabla_{h} y^{h} \rightarrow$ $\left(\bar{y}^{\prime}, \bar{d}_{2}, \bar{d}_{3}\right)$ strongly in $L^{2}\left(\Omega, \mathbb{R}^{3 \times 3}\right), \bar{d}_{k}(0)=e_{k}$ for $k=2,3$ and $\left(\bar{y}, \bar{d}_{2}, \bar{d}_{3}\right) \in \mathcal{A}$.

\section{Properties of $E^{h}$}

We start by using the uniform energy bound of the deformations $\left(y^{h}\right)$, i.e., stationary is not yet needed. Recall the decomposition (32), i.e.,

$$
E^{h}=\mathbb{A}^{h}(x) \operatorname{sym} G^{h}+\frac{1}{h} \zeta^{h}\left(x, h G^{h}\right),
$$

Notice that the uniform bound on $\left|\mathbb{A}^{h}\right| \leq C \beta$ given by (7), the uniform $L^{2}$ bound on $G^{h}$ and the uniform $L^{2}$ bound on the sequence $\left(h^{-1} \zeta^{h}\left(\cdot, h G^{h}\right)\right)_{h>0}$, following from Lemma 3.1, imply a uniform $L^{2}$ bound on the sequence $E^{h}$. Thus $E^{h}$ weakly subconverges to some $E \in L^{2}\left(\Omega, \mathbb{R}^{3 \times 3}\right)$. The frame-indifference (M1) readily implies that $D W^{h}(\cdot, F) F^{T}$ is symmetric for every $F \in \mathbb{R}^{3 \times 3}$ almost everywhere on $\Omega$. For $F=\operatorname{id}_{3 \times 3}+h G^{h}$ the statement skew $\left(D W^{h}(\cdot, F) F^{T}\right)=0$ can be rewritten to

$$
\text { skew }\left(E^{h}\right)=h \text { skew }\left(G^{h}\left(E^{h}\right)^{T}\right) \text {. }
$$

From the uniform $L^{2}$ bound on $E^{h}$ and $G^{h}$ we deduce a uniform $L^{1}$ bound on $\left(h^{-1}\right.$ skew $\left.\left(E^{h}\right)\right)$.

\section{Deriving Euler-Lagrange equations}

Since $\left(y^{h}\right)$ are stationary points of $\mathcal{E}^{h}$ we obtain for any $\psi \in C_{\mathrm{bdy}}^{\infty}\left(\bar{\Omega}, \mathbb{R}^{3}\right)$ the equality

$$
\int_{\Omega}\left(D W^{h}\left(x, \nabla_{h} y^{h}(x)\right): \nabla_{h} \psi(x)-h^{2} g\left(x_{1}\right) \cdot \psi(x)\right) \mathrm{d} x=0 .
$$

By density the equation also holds for arbitrary $\psi \in W_{\text {bdy }}^{1,2}\left(\Omega, \mathbb{R}^{3}\right)$. Using (31) we rewrite this equation to

$$
\int_{\Omega}\left(R^{h} E^{h}: \nabla_{h} \psi-h g \cdot \psi\right) \mathrm{d} x=0 .
$$


For $\psi(x)=\varphi\left(x_{1}\right)$ with $\varphi \in C_{\mathrm{bdy}}^{\infty}\left([0, L], \mathbb{R}^{3}\right)$ the equation (34) reduces to

$$
\int_{0}^{L} \int_{\omega}\left(R^{h} E^{h} e_{1} \cdot \varphi^{\prime}-h g \cdot \varphi\right) \mathrm{d} x^{\prime} \mathrm{d} x_{1}=\int_{0}^{L}\left(R^{h} \bar{E}^{h} e_{1} \cdot \varphi^{\prime}-h g \cdot \varphi\right) \mathrm{d} x_{1}=0
$$

where $\bar{E}^{h}\left(x_{1}\right):=\int_{\omega} E^{h}\left(x_{1}, x^{\prime}\right) \mathrm{d} x^{\prime} \in L^{2}\left((0, L), \mathbb{R}^{3 \times 3}\right)$. Furthermore we denote the first moments with respect to $x_{2}$ and $x_{3}$ of $E$ by $\widetilde{E}, \widehat{E} \in L^{2}\left((0, L), \mathbb{R}^{3 \times 3}\right)$ respectively; more precisely let

$$
\widetilde{E}\left(x_{1}\right)=\int_{\omega} x_{2} E\left(x_{1}, x^{\prime}\right) \mathrm{d} x^{\prime} ; \quad \widehat{E}\left(x_{1}\right)=\int_{\omega} x_{3} E\left(x_{1}, x^{\prime}\right) \mathrm{d} x^{\prime} .
$$

Let $\phi \in C_{\text {bdy }}^{\infty}([0, L])$. Then for $\psi(x)=x_{2} \phi\left(x_{1}\right) R^{h}\left(x_{1}\right) e_{1} \in W_{\text {bdy }}^{1,2}\left(\Omega, \mathbb{R}^{3}\right)$ we obtain

$$
\nabla_{h} \psi(x)=\left(x_{2} \phi^{\prime}\left(x_{1}\right) R^{h}\left(x_{1}\right) e_{1}+x_{2} \phi\left(x_{1}\right)\left(R^{h}\right)^{\prime}\left(x_{1}\right) e_{1}\left|\frac{1}{h} \phi\left(x_{1}\right) R^{h}\left(x_{1}\right) e_{1}\right| 0\right)
$$

and thus (34) simplifies to

$$
\begin{aligned}
0 & =\int_{\Omega}\left(R^{h} E^{h}: \nabla_{h} \psi-h g \cdot \psi\right) \mathrm{d} x \\
& =\int_{0}^{L}\left(R^{h} \widetilde{E}^{h} e_{1} \cdot \phi^{\prime} R^{h} e_{1}+R^{h} \widetilde{E}^{h} e_{1} \cdot \phi\left(R^{h}\right)^{\prime} e_{1}+\frac{1}{h} R^{h} \bar{E}^{h} e_{2} \cdot \phi R^{h} e_{1}\right) \mathrm{d} x_{1} .
\end{aligned}
$$

Introducing $A^{h}:=\left(R^{h}\right)^{T}\left(R^{h}\right)^{\prime}$ this simplifies further to

$$
\int_{0}^{L}\left(\widetilde{E}_{11}^{h} \cdot \phi^{\prime}+\phi \widetilde{E}^{h} e_{1} \cdot A^{h} e_{1}+\phi \frac{1}{h} \bar{E}_{12}^{h}\right) \mathrm{d} x_{1}=0 .
$$

Analogously for $\psi(x)=x_{3} \phi\left(x_{1}\right) R^{h}\left(x_{1}\right) e_{1}$ we get

$$
\int_{0}^{L}\left(\widehat{E}_{11}^{h} \cdot \phi^{\prime}+\phi \widehat{E}^{h} e_{1} \cdot A^{h} e_{1}+\phi \frac{1}{h} \bar{E}_{13}^{h}\right) \mathrm{d} x_{1}=0,
$$

and finally $\psi(x)=x_{3} \phi\left(x_{1}\right) R^{h}\left(x_{1}\right) e_{2}-x_{2} \phi\left(x_{1}\right) R^{h}\left(x_{1}\right) e_{3}$ yields

$$
\int_{0}^{L}\left(\phi^{\prime}\left(\widehat{E}_{21}^{h}-\widetilde{E}_{31}^{h}\right)+\phi\left(\widehat{E}^{h} e_{1} \cdot A^{h} e_{2}-\widetilde{E}^{h} e_{1} \cdot A^{h} e_{3}\right)+\phi \frac{1}{h}\left(\bar{E}_{23}^{h}-\bar{E}_{32}^{h}\right)\right) \mathrm{d} x_{1}=0 .
$$

\section{Consequences of the Euler-Lagrange equations}

Now, by stationary of $\left(y^{h}\right)$, the equation (35) holds for arbitrary $\varphi \in C_{c}^{\infty}\left((0, L), \mathbb{R}^{3}\right)$, and thus

$$
\bar{E}^{h} e_{1}=-h\left(R^{h}\right)^{T} \widehat{g} \quad \text { a.e. in }(0, L),
$$

especially

$$
\bar{E} e_{1}=0 \quad \text { a.e. in }(0, L) .
$$

Furthermore the equations (36), (37) and (38) imply that $\widetilde{E}_{11}^{h}, \widehat{E}_{11}^{h}$ and $\left(\widehat{E}_{21}^{h}-\right.$ $\left.\widetilde{E}_{31}^{h}\right)$ are weakly differentiable. The respective derivatives are in $L^{1}$, as seen by 
combining (33), (39) together with the uniform $L^{2}$ bound on $A^{h}$, which was just $\left(R^{h}\right)^{T}\left(R^{h}\right)^{\prime}$. By Sobolev's Embedding Theorem we thus obtain that

$$
\left(\widetilde{E}_{11}^{h}\right),\left(\widehat{E}_{11}^{h}\right),\left(\widehat{E}_{21}^{h}-\widetilde{E}_{31}^{h}\right) \text { converge strongly in } L^{2}((0, L)) .
$$

From this we immediately get the following: Let $\left(M^{h}\right) \subset L^{2}\left((0, L), \mathbb{R}_{\text {skew }}^{3 \times 3}\right)$ with $M^{h} \rightarrow M \in L^{2}\left((0, L), \mathbb{R}_{\text {skew }}^{3 \times 3}\right)$. Then by direct calculation we obtain

$$
\int_{\Omega} E^{h}: \iota\left(M^{h} \mathfrak{p}\right) \mathrm{d} x=\int_{0}^{L}\left(\widetilde{E}_{31}^{h}-\widehat{E}_{21}^{h}, \widehat{E}_{11}^{h}, \widetilde{E}_{11}^{h}\right) \cdot \operatorname{axl} M^{h} \mathrm{~d} x_{1}
$$

and thus by applying (41) we get

$$
\lim _{h \searrow 0} \int_{\Omega} E^{h}: \iota\left(M^{h} \mathfrak{p}\right) \mathrm{d} x=\lim _{h \searrow 0} \int_{\Omega} E^{h}: \iota(M \mathfrak{p}) \mathrm{d} x .
$$

\section{The limit of the PDE in terms of the stress}

Fix some $\Phi \in C_{\text {bdy }}^{\infty}\left([0, L], \mathbb{R}_{\text {skew }}^{3 \times 3}\right)$ and let $\phi_{1}, \phi_{2}, \phi_{3}$ be given by $\operatorname{axl}(\Phi)=\left(\phi_{1}, \phi_{2}, \phi_{3}\right)$. We then define the test functions

$$
\begin{aligned}
\psi^{h}\left(x_{1}, x_{2}, x_{3}\right) & =R^{h}\left(x_{1}\right) \Phi\left(x_{1}\right) \mathfrak{p}(x) \\
& \left(=x_{3} \phi_{2} R^{h} e_{1}-x_{2} \phi_{3} R^{h} e_{1}+\phi_{1}\left(x_{2} R^{h} e_{3}-x_{3} R^{h} e_{2}\right)\right) .
\end{aligned}
$$

We compute

$$
\nabla_{h} \psi^{h}=\left(R^{h} \Phi^{\prime} \mathfrak{p}+\left(R^{h}\right)^{\prime} \Phi \mathfrak{p}\left|\frac{1}{h} R^{h} \Phi e_{2}\right| \frac{1}{h} R^{h} \Phi e_{3}\right),
$$

and plugging it into (34) we obtain

$$
\begin{aligned}
& \int_{\Omega}\left(R^{h} E^{h}: \nabla_{h} \psi^{h}-h g \cdot \psi^{h}\right) \mathrm{d} x \\
& =\int_{\Omega}\left(E^{h} e_{1} \cdot \Phi^{\prime} \mathfrak{p}+E^{h} e_{1} \cdot A^{h} \Phi \mathfrak{p}+\frac{1}{h} E^{h} e_{2} \cdot \Phi e_{2}+\frac{1}{h} E^{h} e_{3} \cdot \Phi e_{3}\right) \mathrm{d} x .
\end{aligned}
$$

By definition we have $\Phi e_{2}=\phi_{1} e_{3}-\phi_{3} e_{1}$ and $\Phi e_{3}=\phi_{2} e_{1}-\phi_{1} e_{2}$ and thus

$$
\begin{aligned}
E^{h} e_{2} \cdot \Phi e_{2}+E^{h} e_{3} \cdot \Phi e_{3}= & \left(\phi_{1} E_{32}^{h}-\phi_{3} E_{12}^{h}\right)+\left(\phi_{2} E_{13}^{h}-\phi_{1} E_{23}^{h}\right) \\
= & 2 \phi_{1}\left(\text { skew } E^{h}\right)_{32}-2 \phi_{3}\left(\text { skew } E^{h}\right)_{12}+2 \phi_{2}\left(\text { skew } E^{h}\right)_{13} \\
& -\phi_{3} E_{21}^{h}+\phi_{2} E_{31}^{h} \\
= & \left(\Phi: \text { skew } E^{h}\right)+\left(\phi_{2} E_{31}^{h}-\phi_{3} E_{21}^{h}\right)
\end{aligned}
$$

With the preceding calculation it is easy to verify the splitting of (43) into

$$
\int_{\Omega}\left(R^{h} E^{h}: \nabla_{h} \psi^{h}-h g \cdot \psi^{h}\right) \mathrm{d} x=\mathrm{I}^{h}+\mathrm{II}^{h}+\mathrm{III}^{h},
$$


where

$$
\begin{aligned}
\mathrm{I}^{h} & :=\int_{\Omega}\left(E^{h} e_{1} \cdot \Phi^{\prime} \mathfrak{p}\right) \mathrm{d} x=\int_{\Omega} E^{h}: \iota\left(\Phi^{\prime} \mathfrak{p}\right) \mathrm{d} x, \\
\mathrm{II}^{h} & :=\int_{\Omega}\left(\phi_{2} \frac{1}{h} E_{31}^{h}-\phi_{3} \frac{1}{h} E_{21}^{h}\right) \mathrm{d} x, \\
\mathrm{III}^{h} & :=\int_{\Omega}\left(E^{h} e_{1} \cdot A^{h} \Phi \mathfrak{p}+\frac{1}{h} \Phi: \text { skew } E^{h}\right) \mathrm{d} x .
\end{aligned}
$$

The third one will be the most difficult to handle.

Regarding $\mathrm{II}^{h}$, from (39) we obtain $\bar{E}^{h} e_{1}=-h\left(R^{h}\right)^{T} \widehat{g}$ and thus

$$
\mathrm{II}^{h}=\int_{0}^{L}\left(\phi_{3} \widehat{g} \cdot\left(R^{h} e_{2}\right)-\phi_{2} \widehat{g} \cdot\left(R^{h} e_{3}\right)\right) \mathrm{d} x_{1}=\int_{0}^{L} \widehat{g} \cdot\left(R^{h} \Phi e_{1}\right) \mathrm{d} x_{1} .
$$

Regarding III $^{h}$, we claim that we have

$$
\lim _{h \searrow 0} \mathrm{III}^{h}=\lim _{h \searrow 0} \int_{\Omega}\left(E^{h} e_{1} \cdot(A \Phi-\Phi A) \mathfrak{p}\right) \mathrm{d} x .
$$

Indeed, recall that from (12) we have

$$
G^{h}=A^{h} \mathfrak{p} \otimes e_{1}+\left(R^{h}\right)^{T} \nabla_{h} z^{h},
$$

where $z^{h}$ was defined by (13). By making use of (33) we obtain

$$
\begin{aligned}
\frac{1}{h} \text { skew }\left(E^{h}\right) & =\operatorname{skew}\left(G^{h}\left(E^{h}\right)^{T}\right) \\
& =\operatorname{skew}\left(\left(\left(R^{h}\right)^{T} \nabla_{h} z^{h}+A^{h} \mathfrak{p} \otimes e_{1}\right)\left(E^{h}\right)^{T}\right) \\
& =\operatorname{skew}\left(\left(R^{h}\right)^{T} \nabla_{h} z^{h}\left(E^{h}\right)^{T}\right)+\operatorname{skew}\left(\left(A^{h} \mathfrak{p} \otimes e_{1}\right)\left(E^{h}\right)^{T}\right) .
\end{aligned}
$$

Furthermore by the skew-symmetry of $\Phi$ we thus obtain

$$
\frac{1}{h} \Phi: \text { skew }\left(E^{h}\right)=\Phi:\left(\left(R^{h}\right)^{T} \nabla_{h} z^{h}\left(E^{h}\right)^{T}\right)+\Phi:\left(A^{h} \mathfrak{p} \otimes E^{h} e_{1}\right) .
$$

Note that for any $M \in \mathbb{R}^{n \times n}$ and $v, w \in \mathbb{R}^{n}$ we have the algebraic identity

$$
M:(v \otimes w)=\operatorname{tr}\left(M^{T}(v \otimes w)\right)=\operatorname{tr}\left(\left(M^{T} v\right) \otimes w\right)=\left(M^{T} v\right) \cdot w,
$$

which applied to $M=\Phi, v=A^{h} \mathfrak{p}, w=E^{h} e_{1}$ yields for the second term in (49) the equality

$$
\Phi:\left(A^{h} \mathfrak{p} \otimes E^{h} e_{1}\right)=-E^{h} e_{1} \cdot\left(\Phi A^{h} \mathfrak{p}\right) .
$$

With this we can simplify III $^{h}$, given by (46), to

$$
\begin{aligned}
\mathrm{III}^{h} & =\int_{\Omega}\left(E^{h} e_{1} \cdot A^{h} \Phi \mathfrak{p}+\frac{1}{h} \Phi: \text { skew } E^{h}\right) \mathrm{d} x \\
& =\int_{\Omega}\left(E^{h} e_{1} \cdot\left(A^{h} \Phi-\Phi A^{h}\right) \mathfrak{p}+\Phi:\left(\left(R^{h}\right)^{T} \nabla_{h} z^{h}\left(E^{h}\right)^{T}\right)\right) \mathrm{d} x .
\end{aligned}
$$


We start by proving that,

$$
\lim _{h \searrow 0} \int_{\Omega}\left(E^{h} e_{1} \cdot\left(A^{h} \Phi-\Phi A^{h}\right) \mathfrak{p}\right) \mathrm{d} x=\lim _{h \searrow 0} \int_{\Omega}\left(E^{h} e_{1} \cdot(A \Phi-\Phi A) \mathfrak{p}\right) \mathrm{d} x .
$$

which, however, immediately follows from (42) by setting $M^{h}:=A^{h} \Phi-\Phi A^{h}$ and $M:=A \Phi-\Phi A$. If the second term on the right-hand side of (50) vanishes, the claim is proved.

For this we first note that for any $B \in \mathbb{R}^{3 \times 3}$ and $R \in \mathrm{SO}(3)$ we have skew $B=$ skew $\left(R B R^{T}\right)$. This is a straight-forward computation, which relies heavily on the fact, that $3 \times 3$ skew-symmetric matrices have at most two non-vanishing entries per column and row. With $R:=R^{h}$ and $B:=\nabla_{h} z^{h}\left(E^{h}\right)^{T}$ we then obtain

$$
\text { skew }\left(\left(R^{h}\right)^{T} \nabla_{h} z^{h}\left(E^{h}\right)^{T}\right)=\text { skew }\left(\nabla_{h} z^{h}\left(E^{h}\right)^{T}\left(R^{h}\right)^{T}\right)=\text { skew }\left(\nabla_{h} z^{h}\left(R^{h} E^{h}\right)^{T}\right),
$$

and thus

$$
\begin{aligned}
\int_{\Omega} \Phi:\left(\nabla_{h} z^{h}\left(R^{h} E^{h}\right)^{T}\right) \mathrm{d} x & =\int_{\Omega}\left(\left(\nabla_{h} z^{h}\right)^{T} \Phi\right):\left(\left(R^{h} E^{h}\right)^{T}\right) \mathrm{d} x \\
& =-\int_{\Omega} R^{h} E^{h}:\left(\Phi \nabla_{h} z^{h}\right) \mathrm{d} x .
\end{aligned}
$$

We write the right-hand side of the inner product as a gradient and a lower-order term, i.e., $\Phi \nabla_{h} z^{h}=\nabla_{h}\left(\Phi z^{h}\right)-\iota\left(\Phi^{\prime} z^{h}\right)$. Using this identity in (51) we obtain two terms. For the first one we note that $\Phi$ vanishes at the left boundary, and we might use the Euler-Lagrange equation (34) to get

$$
\int_{\Omega}\left(R^{h} E^{h}: \nabla_{h}\left(\Phi z^{h}\right)\right) \mathrm{d} x=h \int_{\Omega} g \cdot\left(\Phi z^{h}\right) \mathrm{d} x \rightarrow 0 .
$$

For the second term we use the strong convergence of $z^{h}$ and $R^{h}$ to go to the limit

$$
\lim _{h \searrow 0} \int_{\Omega}\left(R^{h} E^{h}: \iota\left(\Phi^{\prime} z^{h}\right)\right) \mathrm{d} x=\int_{\Omega}\left(R E e_{1} \cdot\left(\Phi^{\prime} z\right)\right) \mathrm{d} x=\int_{0}^{L}\left(R \bar{E} e_{1} \cdot\left(\Phi^{\prime} z\right)\right) \mathrm{d} x_{1},
$$

where in the last step we used that $R$ and $z$ are independent of $x_{2}, x_{3}$. Since $\bar{E} e_{1}=0$ by (40) this term vanishes as well, and the claim (48) is thus proved.

Inserting (45), (47) and (48) into (44) we obtain

$$
\begin{aligned}
\lim _{h \searrow 0} \int_{\Omega}\left(R^{h} E^{h}: \nabla_{h} \psi-h g \cdot \psi\right) \mathrm{d} x= & \lim _{h \searrow 0} \int_{\Omega} E^{h}: \iota\left((A \Phi-\Phi A) \mathfrak{p}+\Phi^{\prime} \mathfrak{p}\right) \mathrm{d} x \\
& +\lim _{h \searrow 0} \int_{0}^{L} \widehat{g} \cdot\left(R^{h} \Phi e_{1}\right) \mathrm{d} x_{1} .
\end{aligned}
$$

From the strong convergence $R^{h} \rightarrow R$ in $L^{\infty}$ we obtain for the second term

$$
\lim _{h \searrow 0} \int_{0}^{L} \widehat{g} \cdot\left(R^{h} \Phi e_{1}\right) \mathrm{d} x_{1}=\int_{0}^{L} \widehat{g} \cdot\left(R \Phi e_{1}\right) \mathrm{d} x_{1},
$$


while for the first term we will show that

$$
\lim _{h \searrow 0} \int_{\Omega} E^{h}: \iota\left((A \Phi-\Phi A) \mathfrak{p}+\Phi^{\prime} \mathfrak{p}\right) \mathrm{d} x=\frac{\partial \mathcal{K}_{(h)}}{\partial m}\left(m_{d}\right)\left[\left(A \Phi-\Phi A+\Phi^{\prime}\right) \mathfrak{p}\right] .
$$

\section{Identification of the limit}

To show (54) we will first prove the analogue to [5, lemma 3.1], whose approach we will follow from now on.

Lemma 3.2. Let $\left(u^{h}\right) \subset W^{1,2}\left(\Omega, \mathbb{R}^{3}\right)$ be such that $\mathrm{t}\left(u_{2}^{h}, u_{3}^{h}\right) \rightarrow 0$ strongly in $L^{2}$, $\left(\left|\operatorname{sym} \nabla_{h} u^{h}\right|^{2}\right)$ is equi-integrable and $\left(u_{1}^{h}, h u_{2}^{h}, h u_{3}^{h}\right) \rightarrow 0$ strongly in $L^{2}$. Then for all $\phi \in C_{\mathrm{bdy}}^{\infty}([0, L])$ we have

$$
\lim _{h \searrow 0} \int_{\Omega}\left(\phi \mathbb{A}^{h} G^{h}: \nabla_{h} u^{h}\right) \mathrm{d} x=0 .
$$

Proof. Fix some $\phi \in C_{\mathrm{bdy}}^{\infty}([0, L])$ and let $\left(u^{h}\right)$ be as assumed in the lemma. By Prop. A.1 there exists a constant $C_{\omega}>0$, depending only on $\omega$, and sequences $\left(B^{h}\right) \subset W^{1,2}\left((0, L), \mathbb{R}_{\text {skew }}^{3 \times 3}\right),\left(\vartheta^{h}\right) \subset W^{1,2}\left(\Omega, \mathbb{R}^{3}\right)$ and $\left(o^{h}\right) \subset L^{2}\left(\Omega, \mathbb{R}^{3 \times 3}\right)$ with

$$
\operatorname{sym} \nabla_{h} u^{h}=\operatorname{sym} \iota\left(\left(B^{h}\right)^{\prime} \mathfrak{p}\right)+\operatorname{sym} \nabla_{h} \vartheta^{h}+o^{h},
$$

that, in addition, satisfy the bounds

$$
\left\|B^{h}\right\|_{W^{1,2}}+\left\|\vartheta^{h}\right\|_{L^{2}}+\left\|\nabla_{h} \vartheta^{h}\right\|_{L^{2}} \leq C_{\omega}\left\|\operatorname{sym} \nabla_{h} u^{h}\right\|_{L^{2}} .
$$

Furthermore $B^{h}, \vartheta^{h}, o^{h} \rightarrow 0$ strongly in $L^{2}$, and $\left(\left|\left(B^{h}\right)^{\prime}\right|^{2}\right),\left(\left|\nabla_{h} \vartheta^{h}\right|^{2}\right)$ are both equiintegrable. Using (32) we can write (55) as

$$
\begin{aligned}
\int_{\Omega}\left(\phi \mathbb{A}^{h} \operatorname{sym} G^{h}: \operatorname{sym}\left(\nabla_{h} u^{h}\right)\right) \mathrm{d} x= & \int_{\Omega}\left(\phi E^{h}: \operatorname{sym}\left(\nabla_{h} u^{h}\right)\right) \mathrm{d} x \\
& -\frac{1}{h} \int_{\Omega}\left(\phi \zeta^{h}\left(x, h G^{h}\right): \operatorname{sym}\left(\nabla_{h} u^{h}\right)\right) \mathrm{d} x .
\end{aligned}
$$

The first term on the right-hand side can be decomposed with (56) to

$$
\int_{\Omega}\left(\phi E^{h}: \operatorname{sym}\left(\nabla_{h} u^{h}\right)\right) \mathrm{d} x=\int_{\Omega}\left(\phi E^{h}: \operatorname{sym}\left(\iota\left(\left(B^{h}\right)^{\prime} \mathfrak{p}\right)+\nabla_{h} \vartheta^{h}+o^{h}\right)\right) \mathrm{d} x .
$$

Clearly the term containing $o^{h}$ vanishes in the limit. By symmetry of $\mathbb{A}^{h}$ we have skew $E^{h}=\frac{1}{h}$ skew $\zeta^{h}\left(\cdot, h G^{h}\right)$ and thus write

$$
\begin{aligned}
\int_{\Omega} & \left(\phi E^{h}: \operatorname{sym}\left(\iota\left(\left(B^{h}\right)^{\prime} \mathfrak{p}\right)+\nabla_{h} \vartheta^{h}\right)\right) \mathrm{d} x \\
= & \int_{\Omega}\left(\phi E^{h}:\left(\iota\left(\left(B^{h}\right)^{\prime} \mathfrak{p}\right)+\nabla_{h} \vartheta^{h}\right)\right) \mathrm{d} x \\
& \quad-\int_{\Omega}\left(\phi \frac{1}{h} \zeta^{h}\left(x, h G^{h}\right): \text { skew }\left(\iota\left(\left(B^{h}\right)^{\prime} \mathfrak{p}\right)+\nabla_{h} \vartheta^{h}\right)\right) \mathrm{d} x .
\end{aligned}
$$


Combining (58) with (59) yields

$$
\begin{aligned}
\lim _{h \searrow 0} \int_{\Omega}\left(\phi \mathbb{A}^{h} G^{h}: \operatorname{sym}\left(\nabla_{h} u^{h}\right)\right) \mathrm{d} x & =\lim _{h \searrow 0} \int_{\Omega}\left(\phi E^{h}:\left(\iota\left(\left(B^{h}\right)^{\prime} \mathfrak{p}\right)+\nabla_{h} \vartheta^{h}\right)\right) \mathrm{d} x \\
& -\lim _{h \searrow 0} \int_{\Omega} \phi \frac{1}{h} \zeta^{h}\left(x, h G^{h}\right):\left(\iota\left(\left(B^{h}\right)^{\prime} \mathfrak{p}\right)+\nabla_{h} \vartheta^{h}\right) \mathrm{d} x .
\end{aligned}
$$

We start with the first term on the right-hand side, i.e.,

$$
\int_{\Omega}\left(\phi E^{h}: \iota\left(\left(B^{h}\right)^{\prime} \mathfrak{p}\right)\right) \mathrm{d} x
$$

By applying (42) with $M^{h}=\left(B^{h}\right)^{\prime}$ and $M=0$ we see that this term vanishes in the limit. Secondly we study

$$
\int_{\Omega}\left(\phi E^{h}: \nabla_{h} \vartheta^{h}\right) \mathrm{d} x
$$

and for this we rewrite the term to

$$
\begin{aligned}
\int_{\Omega}\left(\phi E^{h}: \nabla_{h} \vartheta^{h}\right) \mathrm{d} x & =\int_{\Omega}\left(\phi R^{h} E^{h}: R^{h} \nabla_{h} \vartheta^{h}\right) \mathrm{d} x \\
& =\int_{\Omega}\left(R^{h} E^{h}: \nabla_{h}\left(R^{h} \phi \vartheta^{h}\right)\right) \mathrm{d} x-\int_{\Omega}\left(R^{h} E^{h}: \iota\left(\left(R^{h} \phi\right)^{\prime} \vartheta^{h}\right)\right) \mathrm{d} x .
\end{aligned}
$$

For the first term on the right-hand side we use the Euler-Lagrange equation and obtain

$$
\int_{\Omega} E^{h}: \nabla_{h}\left(R^{h} \phi \vartheta^{h}\right) \mathrm{d} x=h \int_{\Omega} g \cdot\left(R^{h} \phi \vartheta^{h}\right) \rightarrow 0,
$$

while we split once more the second term on the right-hand side of (600) into

$$
\begin{aligned}
\int_{\Omega}\left(R^{h} E^{h}: \iota\left(\left(R^{h} \phi\right)^{\prime} \vartheta^{h}\right)\right) \mathrm{d} x= & \int_{\Omega}\left(R^{h} E^{h} e_{1} \cdot\left(R^{h} \phi\right)^{\prime}\left(\vartheta^{h}-\bar{\vartheta}^{h}\right)\right) \mathrm{d} x \\
& +\int_{\Omega}\left(R^{h} E^{h} e_{1} \cdot\left(R^{h} \phi\right)^{\prime} \bar{\vartheta}^{h}\right) \mathrm{d} x,
\end{aligned}
$$

where $\bar{\vartheta}^{h}\left(x_{1}\right)=\int_{\omega} \vartheta\left(x_{1}, x^{\prime}\right) \mathrm{d} x$. By the uniform bound of $h\left(R^{h}\right)^{\prime \prime}$ in $L^{2}$, stated in (10), we obtain the uniform bound of $h\left(R^{h}\right)^{\prime}$ in $W^{1,2}$. From the compact Sobolev embedding we obtain that $\left(h\left(R^{h}\right)^{\prime}\right)$ is strongly compact in $L^{\infty}$. Since $\left(R^{h}\right)^{\prime}$ is bounded in $L^{2}$, we have $h\left(R^{h}\right)^{\prime} \rightarrow 0$ strongly in $L^{2}$. By uniqueness of the limit we have $h\left(R^{h}\right)^{\prime} \rightarrow 0$ strongly in $L^{\infty}$. We apply Poincaré's inequality and obtain

$$
\left\|\vartheta^{h}-\bar{\vartheta}^{h}\right\|_{L^{2}} \leq C\left\|\partial_{2} \vartheta^{h}\right\|_{L^{2}} \leq C h\left\|\nabla_{h} \vartheta^{h}\right\|_{L^{2}} \leq C h .
$$

This bound, together with $h\left(R^{h}\right)^{\prime} \rightarrow 0$ strongly in $L^{\infty}$, implies

$$
h\left(R^{h} \phi\right)^{\prime} \frac{\left(\vartheta^{h}-\bar{\vartheta}^{h}\right)}{h} \rightarrow 0 \quad \text { strongly in } L^{2}
$$


while for the second term in (61) we use Sobolev embedding to obtain $\bar{\vartheta}^{h} \rightarrow 0$ in $L^{\infty}$. Combining both we conclude the vanishing of (61). Finally for the last remaining term in (57), namely

$$
\lim _{h \searrow 0} \int_{\Omega} \phi\left(x_{1}\right) \frac{1}{h} \zeta^{h}\left(x, h G^{h}\right):\left(\iota\left(\left(B^{h}\right)^{\prime} \mathfrak{p}\right)+\nabla_{h} \vartheta^{h}\right) \mathrm{d} x,
$$

we use that $\left(\left|\left(B^{h}\right)^{\prime}\right|^{2}\right)$ and $\left(\left|\nabla_{h} \vartheta^{h}\right|^{2}\right)$ are equi-integrable, and thus by virtue of Lemma 3.1 this term vanishes as well. This finishes the proof of the lemma.

We finally prove (54). For this we decompose $E^{h}$ into $E^{h}=\mathbb{A}^{h} G^{h}+\frac{1}{h} \zeta^{h}\left(\cdot, h G^{h}\right)$ and apply Lemma 3.1 to obtain

$$
\begin{aligned}
\lim _{h \searrow 0} \int_{\Omega} E^{h}: & \iota\left((A \Phi-\Phi A) \mathfrak{p}+\Phi^{\prime} \mathfrak{p}\right) \mathrm{d} x \\
& =\lim _{h \searrow 0} \int_{\Omega} \mathbb{A}^{h} G^{h}: \iota\left((A \Phi-\Phi A) \mathfrak{p}+\Phi^{\prime} \mathfrak{p}\right) \mathrm{d} x .
\end{aligned}
$$

From the decomposition (18) we get

$$
\operatorname{sym} G^{h}=\operatorname{sym} \iota\left(m_{d}\right)+\operatorname{sym} \nabla_{h} \psi^{h}+o^{h}
$$

for the fixed part $m_{d} \in L^{2}\left(\Omega, \mathbb{R}^{3}\right)$ and the corrector sequence $\psi^{h}$ introduced in (15) and (16) respectively, and the sequence $o^{h}$, which converges strongly to zero in $L^{2}$. We show that sym $\nabla_{h} \psi^{h}$ and $\operatorname{sym} \nabla_{h} \psi_{m_{d}}^{h}$ are, up to $L^{2}$-concentration, close in $L^{2}$, where $\left(\psi_{m_{d}}^{h}\right)$ is the relaxation sequence given by Lemma 2.2. Indeed, we first use identity (17) to obtain

$$
\operatorname{sym} \nabla_{h} \psi^{h}=\operatorname{sym} \iota\left(\left(\Psi^{h}\right)^{\prime} \mathfrak{p}\right)+\operatorname{sym} \nabla_{h} v^{h}
$$

where $\Psi^{h}, v^{h}$ are defined prior to this decomposition. By applying [14, lemma 2.17] to $\left(\Psi^{h}\right)$ and $\left(v^{h}\right)$, we obtain a subsequence $(h)$ (not relabeled), a sequence of measurable sets $O^{h}$ with $\lim _{h \backslash 0} \mathcal{L}^{3}\left(\Omega \backslash O^{h}\right)=0$ and a sequences $\left(\widetilde{\Psi}^{h}\right),\left(\widetilde{v}^{h}\right)$ such that $\left(\left|\left(\widetilde{\Psi}^{h}\right)^{\prime}\right|^{2},\left|\left(\nabla_{h} \widetilde{v}^{h}\right)\right|^{2}\right.$ are equi-integrable and

$$
\left\|\left(\Psi^{h}-\widetilde{\Psi}^{h}\right)^{\prime}\right\|_{L^{2}\left(O^{h}\right)}+\left\|\nabla_{h}\left(v^{h}-\widetilde{v}^{h}\right)\right\|_{L^{2}\left(O^{h}\right)} \rightarrow 0 .
$$

By (A.2) there exists $\left(\widetilde{\psi}^{h}\right) \subset W^{1,2}\left(\Omega, \mathbb{R}^{3}\right)$ such that

$$
\operatorname{sym} \nabla_{h} \widetilde{\psi}^{h}=\operatorname{sym} \iota\left(\left(\widetilde{\Psi}^{h}\right)^{\prime} \mathfrak{p}\right)+\operatorname{sym} \nabla_{h} \widetilde{v}^{h} .
$$

By construction it satisfies

$$
\left(\left|\operatorname{sym} \nabla_{h} \widetilde{\psi}^{h}\right|^{2}\right) \text { is equi-integrable, and } \lim _{h \searrow 0}\left\|\operatorname{sym}\left(\nabla_{h} \psi^{h}-\nabla_{h} \widetilde{\psi}^{h}\right)\right\|_{L^{2}\left(O^{h}\right)}=0 .
$$

Furthermore, for any $0<a<L$ we decompose the domain of integration and obtain

$$
\begin{aligned}
& \left\|\operatorname{sym} \nabla_{h}\left(\widetilde{\psi}^{h}-\psi_{m_{d}}^{h}\right)\right\|_{L^{2}(\Omega)}^{2} \\
& =\left\|\operatorname{sym} \nabla_{h}\left(\widetilde{\psi}^{h}-\psi_{m_{d}}^{h}\right)\right\|_{L^{2}((0, a) \times \omega)}^{2}+\left\|\operatorname{sym} \nabla_{h}\left(\widetilde{\psi}^{h}-\psi_{m_{d}}^{h}\right)\right\|_{L^{2}((a, L) \times \omega)}^{2} .
\end{aligned}
$$


For the second term on the right-hand side we use use the coercivity of $Q^{h}$ to obtain

$$
\alpha\left\|\operatorname{sym} \nabla_{h}\left(\widetilde{\psi}^{h}-\psi_{m_{d}}^{h}\right)\right\|_{L^{2}((a, L) \times \omega)}^{2} \leq \frac{1}{2} \int_{(a, L) \times \omega} \mathbb{A}^{h} \nabla_{h}\left(\widetilde{\psi}^{h}-\psi_{m_{d}}^{h}\right): \nabla_{h}\left(\widetilde{\psi}^{h}-\psi_{m_{d}}^{h}\right) .
$$

Let $\rho \in C^{\infty}([0, L])$ be a cut-off function such that $\rho \geq 0, \rho=0$ on $[0, a / 2]$ and $\rho=1$ on $[a, L]$. With this we calculate

$$
\begin{aligned}
\alpha\left\|\operatorname{sym} \nabla_{h}\left(\widetilde{\psi}^{h}-\psi_{m_{d}}^{h}\right)\right\|_{L^{2}((a, L) \times \omega)}^{2} \leq & \frac{1}{2} \int_{\Omega} \rho \mathbb{A}^{h} \nabla_{h}\left(\widetilde{\psi}^{h}-\psi_{m_{d}}^{h}\right): \nabla_{h}\left(\widetilde{\psi}^{h}-\psi_{m_{d}}^{h}\right) \\
= & \frac{1}{2} \int_{\Omega} \rho \mathbb{A}^{h}\left(\iota\left(m_{d}\right)+\nabla_{h} \widetilde{\psi}^{h}\right): \nabla_{h}\left(\widetilde{\psi}^{h}-\psi_{m_{d}}^{h}\right) \\
& -\frac{1}{2} \int_{\Omega} \rho \mathbb{A}^{h}\left(\iota\left(m_{d}\right)+\nabla_{h} \psi_{m_{d}}^{h}\right): \nabla_{h}\left(\widetilde{\psi}^{h}-\psi_{m_{d}}^{h}\right) .
\end{aligned}
$$

The second term vanishes by virtue of Lemma 2.2, while for the second one we use the decomposition (18), i.e.,

$$
\operatorname{sym} G^{h}=\operatorname{sym} \iota\left(m_{d}\right)+\operatorname{sym} \nabla_{h} \psi^{h}+o^{h},
$$

to write

$$
\begin{aligned}
\int_{\Omega} \rho \mathbb{A}^{h}\left(\iota\left(m_{d}\right)+\nabla_{h} \widetilde{\psi}^{h}\right): \nabla_{h}\left(\widetilde{\psi}^{h}-\psi_{m_{d}}^{h}\right) & =\int_{\Omega} \rho \mathbb{A}^{h}\left(G^{h}+o^{h}\right): \nabla_{h}\left(\widetilde{\psi}^{h}-\psi_{m_{d}}^{h}\right) \\
& +\int_{\Omega} \rho \mathbb{A}^{h}\left(\nabla_{h}\left(\widetilde{\psi}^{h}-\psi^{h}\right)\right): \nabla_{h}\left(\widetilde{\psi}^{h}-\psi_{m_{d}}^{h}\right) .
\end{aligned}
$$

The sequence $o^{h}$ converges strongly to 0 and thus the term containing it vanishes in the limit. By Prop. 2.2 the sequence $\left|\operatorname{sym}\left(\nabla_{h} \psi_{m_{d}}^{h}\right)\right|^{2}$ and, by construction, the sequence $\left|\operatorname{sym}\left(\nabla_{h} \widetilde{\psi}^{h}\right)\right|^{2}$ are both equi-integrable. Thus by applying Lemma 3.2 the first term vanishes. For the second one we decompose $\Omega=O^{h} \cup\left(\Omega \backslash O^{h}\right)$ and estimate with Hölder's inequality

$$
\begin{aligned}
& \left|\int_{\Omega} \rho \mathbb{A}^{h} \nabla_{h}\left(\widetilde{\psi}^{h}-\psi^{h}\right): \nabla_{h}\left(\widetilde{\psi}^{h}-\psi_{m_{d}}^{h}\right)\right| \\
& \leq \beta\left|\int_{\Omega} \operatorname{sym} \nabla_{h}\left(\widetilde{\psi}^{h}-\psi^{h}\right): \operatorname{sym} \nabla_{h}\left(\widetilde{\psi}^{h}-\psi_{m_{d}}^{h}\right)\right| \\
& \leq \beta\left\|\operatorname{sym} \nabla_{h}\left(\widetilde{\psi}^{h}-\psi^{h}\right)\right\|_{L^{2}\left(O^{h}\right)}\left\|\operatorname{sym} \nabla_{h}\left(\widetilde{\psi}^{h}-\psi_{m_{d}}^{h}\right)\right\|_{L^{2}(\Omega)} \\
& \quad+\beta\left\|\operatorname{sym} \nabla_{h}\left(\widetilde{\psi}^{h}-\psi^{h}\right)\right\|_{L^{2}(\Omega)}\left\|\operatorname{sym} \nabla_{h}\left(\widetilde{\psi}^{h}-\psi_{m_{d}}^{h}\right)\right\|_{L^{2}\left(\Omega \backslash O^{h}\right)} .
\end{aligned}
$$

First note that

$$
\left\|\operatorname{sym} \nabla_{h}\left(\widetilde{\psi}^{h}-\psi_{m_{d}}^{h}\right)\right\|_{L^{2}(\Omega)}, \quad\left\|\operatorname{sym} \nabla_{h}\left(\widetilde{\psi}^{h}-\psi^{h}\right)\right\|_{L^{2}(\Omega)}
$$

are uniformly bounded in $h$. Furthermore utilizing (63) we obtain that

$$
\lim _{h \searrow 0}\left\|\operatorname{sym} \nabla_{h}\left(\widetilde{\psi}^{h}-\psi^{h}\right)\right\|_{L^{2}\left(O^{h}\right)}=0
$$


and thus the first term on the right-hand side vanishes. For the second term on the right-hand side of (65) we apply the equi-integrability of $\left(\left|\operatorname{sym} \nabla_{h}\left(\widetilde{\psi}^{h}-\psi_{m_{d}}^{h}\right)\right|^{2}\right)$ together with $\mathcal{L}^{3}\left(\Omega \backslash O^{h}\right) \rightarrow 0$ for $h \searrow 0$, and obtain that the term vanishes as well. Returning to (64), we take a sequence $a=a(h)$ with $a(h) \searrow 0$ for $h \searrow 0$ such that

$$
\lim _{h \searrow 0}\left\|\operatorname{sym} \nabla_{h}\left(\widetilde{\psi}^{h}-\psi_{m_{d}}^{h}\right)\right\|_{L^{2}((a(h), L) \times \omega)}=0 .
$$

By equi-integrability we also obtain

$$
\lim _{h \backslash 0}\left\|\operatorname{sym} \nabla_{h}\left(\widetilde{\psi}^{h}-\psi_{m_{d}}^{h}\right)\right\|_{L^{2}((0, a(h)) \times \omega)}=0,
$$

and thus

$$
\lim _{h \searrow 0}\left\|\operatorname{sym} \nabla_{h}\left(\widetilde{\psi}^{h}-\psi_{m_{d}}^{h}\right)\right\|_{L^{2}(\Omega)}=0 .
$$

Returning to (62), we first approximate $\operatorname{sym} \nabla_{h} \psi^{h}$ by sym $\nabla_{h} \widetilde{\psi}^{h}$, and then the latter by $\operatorname{sym} \nabla_{h} \psi_{m_{d}}^{h}$, thus obtaining

$$
\begin{aligned}
& \lim _{h \searrow 0} \int_{\Omega} E^{h}: \iota\left((A \Phi-\Phi A) \mathfrak{p}+\Phi^{\prime} \mathfrak{p}\right) \mathrm{d} x \\
& \quad=\lim _{h \searrow 0} \int_{\Omega} \mathbb{A}^{h} G^{h}: \iota\left((A \Phi-\Phi A) \mathfrak{p}+\Phi^{\prime} \mathfrak{p}\right) \mathrm{d} x \\
& \quad=\lim _{h \searrow 0} \int_{\Omega} \mathbb{A}^{h}\left(\iota\left(m_{d}\right)+\nabla_{h} \psi_{m_{d}}^{h}\right): \iota\left((A \Phi-\Phi A) \mathfrak{p}+\Phi^{\prime} \mathfrak{p}\right) \mathrm{d} x \\
& \quad=\frac{\partial \mathcal{K}_{(h)}}{\partial m}\left(m_{d}\right)\left[\left(A \Phi-\Phi A+\Phi^{\prime}\right) \mathfrak{p}\right] .
\end{aligned}
$$

We combine (52), (53) and (66) $)$, obtaining

$$
\begin{aligned}
0=\lim _{h \searrow 0} \int_{\Omega}\left(R^{h} E^{h}: \nabla_{h} \psi-h \widehat{g} \cdot \partial_{1} \psi\right) \mathrm{d} x= & \frac{\partial \mathcal{K}_{(h)}}{\partial m}\left(m_{d}\right)\left[\left(A \Phi-\Phi A+\Phi^{\prime}\right) \mathfrak{p}\right] \\
& -\int_{0}^{L} \widehat{g} \cdot\left(R \Phi e_{1}\right) \mathrm{d} x_{1} .
\end{aligned}
$$

If

$$
\frac{\partial \mathcal{K}_{(h)}}{\partial m}\left(m_{d}\right)\left[\left(A \Phi-\Phi A+\Phi^{\prime}\right) \mathfrak{p}\right]=\left(\frac{\partial}{\partial B} \mathcal{K}_{(h)}^{0}\right)(A)\left[A \Phi-\Phi A+\Phi^{\prime}\right]
$$

holds, then (67) reads

$$
\left(\frac{\partial}{\partial B} \mathcal{K}_{(h)}^{0}\right)(A)\left[A \Phi-\Phi A+\Phi^{\prime}\right]=\int_{0}^{L} \widehat{g} \cdot\left(R \Phi e_{1}\right) \mathrm{d} x_{1},
$$

and by Lemma 2.4 this is equivalent to $\left(\bar{y}, \bar{d}_{2}, \bar{d}_{3}\right)$ being a stationary point of $\mathcal{E}^{0}$. After replacing both sides in (68) by the more explicit representations (22) and (29), we see that it suffices to show that the fixed part $m_{d}$ is given by $m\left(A, b_{\min }(A)\right)$. By 
definition of $m_{d}$ in (15) we have $m_{d}=m\left(A, p_{1}\right)$ where $p_{1}$ is some $L^{2}$ function. By the characterization given in (25) the equality $p_{1}=b_{\min }(A)$ follows, if

$$
\frac{\partial \mathcal{K}_{(h)}(m(A, \cdot))}{\partial b}\left(p_{1}\right)[\mu]=0 \quad \text { for all } \mu \in L^{2}((0, L)) .
$$

Using (24) we see that this is equivalent to

$$
\left.\lim _{h \searrow 0} \int_{\Omega} \mathbb{A}^{h}\left(\iota\left(m_{d}\right)\right)+\operatorname{sym} \nabla_{h} \psi_{m_{d}}^{h}\right): \iota\left(\mu e_{1}\right) \mathrm{d} x=0 \quad \text { for all } \mu \in L^{2}((0, L)) .
$$

Similar to before we can replace $\operatorname{sym} \nabla_{h} \psi_{m_{d}}^{h}$ by $\operatorname{sym} \nabla_{h} \psi^{h}$. Then we can approximate $\mathbb{A}^{h} G^{h}$ by $E^{h}$ with Lemma 3.1, and the statement (69) is then seen to be equivalent to

$$
\lim _{h \searrow 0} \int_{0}^{L} \bar{E}_{11}^{h} \mu \mathrm{d} x=0 \quad \text { for all } \mu \in L^{2}((0, L)),
$$

which now easily follows from (40).

Acknowledgment: The author thanks his $\mathrm{PhD}$ advisor Peter Hornung as well as Igor Velčić for many valuable discussions, encouragement and general support. The author was supported by DFG under Grant agreement No. HO4697/1-1.

\section{A. Appendix}

For convenience of the reader we recall a type of decomposition introduced in [10, 11]. More precisely the variant proved in [14, corollary 2.3, lemma 2.4]

Proposition A.1. Let $L>0$ and $\Omega=(0, L) \times \omega$, where $\omega$ is an open, connected bounded Lipschitz-domain, which is centered at the origin in the sense of (6). Let $\left(u^{h}\right) \subset W^{1,2}\left(\Omega, \mathbb{R}^{3}\right)$ with $\mathrm{t}\left(u_{2}^{h}, u_{3}^{h}\right) \rightarrow 0$ in $L^{2}((0, L))$,

$$
\sup _{h>0}\left\|\operatorname{sym} \nabla_{h} u^{h}\right\|_{L^{2}}<\infty \quad \text { and } \quad\left(u_{1}^{h}, h u_{2}^{h}, h u_{3}^{h}\right) \rightarrow 0 \text { strongly in } L^{2}\left(\Omega, \mathbb{R}^{3}\right) .
$$

Then there exists a constant $C_{\omega}>0$, depending only on $\omega$, and sequences $\left(B^{h}\right) \subset$ $W^{1,2}\left((0, L), \mathbb{R}_{\text {skew }}^{3 \times 3}\right),\left(\vartheta^{h}\right) \subset W^{1,2}\left(\Omega, \mathbb{R}^{3}\right)$ and $\left(o^{h}\right) \subset L^{2}\left(\Omega, \mathbb{R}^{3 \times 3}\right)$ with

$$
\operatorname{sym} \nabla_{h} u^{h}=\operatorname{sym} \iota\left(\left(B^{h}\right)^{\prime} \mathfrak{p}\right)+\operatorname{sym} \nabla_{h} \vartheta^{h}+o^{h},
$$

and satisfy the bounds

$$
\left\|B^{h}\right\|_{W^{1,2}}+\left\|\vartheta^{h}\right\|_{L^{2}}+\left\|\nabla_{h} \vartheta^{h}\right\|_{L^{2}} \leq C_{\omega}\left\|\operatorname{sym} \nabla_{h} u^{h}\right\|_{L^{2}} .
$$

Furthermore $B^{h}, o^{h}, \vartheta^{h} \rightarrow 0$ strongly in $L^{2}$. If, in addition, $\left(\left|\operatorname{sym} \nabla_{h} u^{h}\right|^{2}\right)$ is equiintegrable, then so are $\left(\left|\left(B^{h}\right)^{\prime}\right|^{2}\right)$ and $\left(\left|\nabla_{h} \vartheta^{h}\right|^{2}\right)$.

The reverse holds true as well: 
Proposition A.2. Let $L>0$ and $\Omega=(0, L) \times \omega$, where $\omega$ is an open, connected bounded Lipschitz-domain, which is centered at the origin in the sense of (6). Let $\left(B^{h}\right) \subset W^{1,2}\left((0, L), \mathbb{R}_{\text {skew }}^{3 \times 3}\right),\left(\vartheta^{h}\right) \subset W^{1,2}\left(\Omega, \mathbb{R}^{3}\right)$ be sequences with $B^{h} \rightarrow 0$ strongly in $L^{2}\left((0, L), \mathbb{R}^{3 \times 3}\right)$ and $\vartheta^{h} \rightarrow 0$ strongly in $L^{2}\left(\Omega, \mathbb{R}^{3}\right)$. Then there exists $\left(u^{h}\right) \subset$ $W^{1,2}\left(\Omega, \mathbb{R}^{3}\right)$ with $\mathrm{t}\left(u_{2}^{h}, u_{3}^{h}\right) \rightarrow 0$ in $L^{2}((0, L))$ and

$$
\left(u_{1}^{h}, h u_{2}^{h}, h u_{3}^{h}\right) \rightarrow 0 \text { strongly in } L^{2}\left(\Omega, \mathbb{R}^{3}\right)
$$

such that

$$
\operatorname{sym} \nabla_{h} u^{h}=\operatorname{sym} \iota\left(\left(B^{h}\right)^{\prime} \mathfrak{p}\right)+\operatorname{sym} \nabla_{h} \vartheta^{h} .
$$




\section{References}

[1] J. M. Ball. Minimizers and the Euler-Lagrange equations. In Trends and applications of pure mathematics to mechanics (Palaiseau, 1983), volume 195 of Lecture Notes in Phys., pages 1-4. Springer, Berlin, 1984.

[2] John M. Ball. Some open problems in elasticity. In Geometry, mechanics, and dynamics, pages 3-59. Springer, New York, 2002.

[3] James Bernoulli. Quadratura curvae, e cujus evolutione describitur inflexae laminae curvatura. In Die Werke von Jakob Bernoulli, pages 223-227. Birkhäuser, 1692. Med. CLXX; Ref. UB: L Ia 3, p 211-212.

[4] A. Braides, I. Fonseca, and G. Francfort. 3D-2D asymptotic analysis for inhomogeneous thin films. Indiana Univ. Math. J., 49(4):1367-1404, 2000.

[5] Mario Bukal, Matthäus Pawelczyk, and Igor Velčić. Derivation of homogenized Euler-Lagrange equations for von Kármán rods. J. Differential Equations, 262(11):5565-5605, 2017.

[6] Elisa Davoli and Maria Giovanna Mora. Convergence of equilibria of thin elastic rods under physical growth conditions for the energy density. Proc. Roy. Soc. Edinburgh Sect. A, 142(3):501-524, 2012.

[7] Leonhard Euler. Methodus inveniendi lineas curvas maximi minimive proprietate gaudentes, sive solutio problematis isoperimetrici lattissimo sensu accepti, chapter Additamentum 1. eulerarchive.org E065, 1744.

[8] Gero Friesecke, Richard D. James, and Stefan Müller. A theorem on geometric rigidity and the derivation of nonlinear plate theory from three-dimensional elasticity. Comm. Pure Appl. Math., 55(11):1461-1506, 2002.

[9] Gero Friesecke, Richard D. James, and Stefan Müller. A hierarchy of plate models derived from nonlinear elasticity by gamma-convergence. Arch. Ration. Mech. Anal., 180(2):183-236, 2006.

[10] Georges Griso. Asymptotic behavior of structures made of curved rods. Anal. Appl. (Singap.), 6(1):11-22, 2008.

[11] Georges Griso. Decompositions of displacements of thin structures. J. Math. Pures Appl. (9), 89(2):199-223, 2008.

[12] Hervé Le Dret and Annie Raoult. The nonlinear membrane model as variational limit of nonlinear three-dimensional elasticity. J. Math. Pures Appl. (9), 74(6):549-578, 1995.

[13] Raph Levien. The elastica: a mathematical history. Technical Report UCB/EECS-2008-103, EECS Department, University of California, Berkeley, Aug 2008. 
[14] Maroje Marohnić and Igor Velčić. Non-periodic homogenization of bendingtorsion theory for inextensible rods from 3D elasticity. Ann. Mat. Pura Appl. (4), 195(4):1055-1079, 2016.

[15] M. G. Mora and S. Müller. Convergence of equilibria of three-dimensional thin elastic beams. Proc. Roy. Soc. Edinburgh Sect. A, 138(4):873-896, 2008.

[16] M. G. Mora, S. Müller, and M. G. Schultz. Convergence of equilibria of planar thin elastic beams. Indiana Univ. Math. J., 56(5):2413-2438, 2007.

[17] Maria Giovanna Mora and Stefan Müller. Derivation of the nonlinear bendingtorsion theory for inextensible rods by $\Gamma$-convergence. Calc. Var. Partial Differential Equations, 18(3):287-305, 2003.

[18] Stefan Neukamm. Homogenization, linearization and dimension reduction in elasticity with variational methods. $\mathrm{PhD}$ thesis, Technische Universität München, 2010.

[19] Igor Velčić. On the general homogenization of von Kármán plate equations from three-dimensional nonlinear elasticity. Anal. Appl. (Singap.), 15(1):1-49, 2017. 